This item was submitted to Loughborough's Research Repository by the author.

Items in Figshare are protected by copyright, with all rights reserved, unless otherwise indicated.

\title{
Visual grammar in practice: negotiating the arrangement of speech bubbles in storyboards
}

\section{PLEASE CITE THE PUBLISHED VERSION}

http://dx.doi.org/10.1515/sem-2013-0008

\section{PUBLISHER}

Walter de Gruyter and International Association for Semiotic Studies

VERSION

VoR (Version of Record)

LICENCE

CC BY-NC-ND 4.0

\section{REPOSITORY RECORD}

Greiffenhagen, Christian. 2019. "Visual Grammar in Practice: Negotiating the Arrangement of Speech Bubbles in Storyboards". figshare. https://hdl.handle.net/2134/14194. 
This item was submitted to Loughborough's Institutional Repository (https://dspace.lboro.ac.uk/) by the author and is made available under the following Creative Commons Licence conditions.

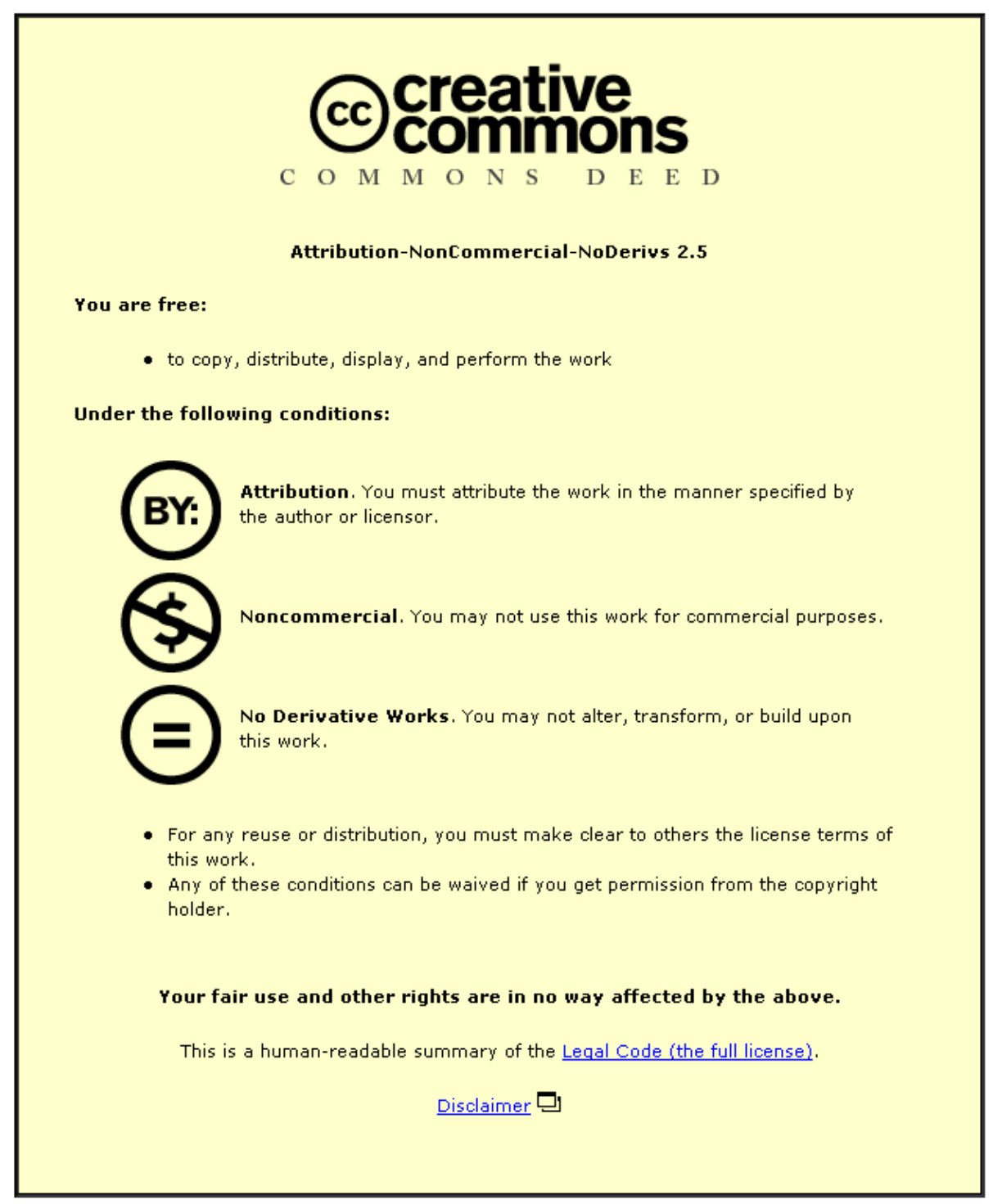

For the full text of this licence, please go to: http://creativecommons.org/licenses/by-nc-nd/2.5/ 


\title{
Christian Greiffenhagen
}

\section{Visual grammar in practice: Negotiating the arrangement of speech bubbles in storyboards*}

\begin{abstract}
It is generally acknowledged that we live in an increasingly visual culture, populated with a variety of visual objects. Researchers have recently started to investigate the underlying regularities, the "visual grammar," according to which these objects are assembled. While most existing studies base their analysis on products (such as advertisements, movies or pages from newspapers), this paper studies the processes through which such products are assembled, thereby investigating visual grammar in practice.

The particular objects analyzed are storyboards that were produced by secondary school pupils using a new computerized storyboarding tool as part of their engagement with Shakespeare's Macbeth. The paper focuses on situations in which pupils explicitly discuss and negotiate the placement of speech bubbles, thereby revealing aspects of the "meaning-effects" of such placements.
\end{abstract}

Keywords: visual grammar; visual culture; multimodality; storyboards; video analysis; ethnomethodology

Christian Greiffenhagen: University of Nottingham.

E-mail: christian.greiffenhagen@nottingham.ac.uk

\footnotetext{
* I am most indebted to the two teachers and their pupils who helped me with this project by allowing me to spend an extensive period of time in their classrooms. Without their generosity this study could not have been conducted. I am very grateful for the invaluable technical support of Peter Blore (Graphic Support Workshop, University of Manchester) in the digital rendering of pupils' hands from the video frames. I would also like to thank Mike Ball, Phil Brooker, Jacqueline Eke, Jon Hindmarsh, Jonas Ivarsson, Carey Jewitt, Lorenza Mondada, Greg Smith, Wes Sharrock, and Alexandra Weilenmann for helpful comments and criticisms on earlier versions of this paper. Part of this research was supported through a British Academy Postdoctoral Fellowship and a Simon Research Fellowship (funded through an endowment made to the University of Manchester).
} 


\section{Introduction}

It is generally acknowledged that we live in an increasingly visual culture (e.g., Evans and Hall 1999; Sturken and Cartwright 2001; Mirzoeff 2009 [1999]). As a result of a variety of technological developments, in particular the vast expansion of electronic media and ubiquitous presence of screens and other displays, our world is now saturated with all kinds of visual images (in the form of magazines, television programs, movies, games, etc.). Despite this, social science researchers have tended to neglect the visual dimensions of the social world. As Sturken and Cartwright observe: "It is a paradox of the twentieth century that while visual images have increasingly come to dominate our culture, our colleges and universities traditionally have devoted relatively little attention to visual media" (2001:4). It is only recently that the campaign for a "visual turn" in the social sciences has gained momentum (e.g., Emmison and Smith 2000; Banks 2001; Sturken and Cartwright 2001; van Leeuwen and Jewitt 2001; Pink 2006; Rose 2007 [2001]; Stanczak 2007).

In one line of inquiry, researchers have started to explore the norms and conventions according to which visual objects are composed and experienced. This kind of research has been done predominantly on the basis of finished products. From the early seminal studies - such as Hall's (1972) "The determinations of news photographs," Williamson's (1978) Decoding Advertisements, and Goffman's (1979) Gender Advertisements - to more recent studies, the emphasis has been on products and not processes. That is to say, researchers have typically taken visual objects (such as news photographs or advertisements) as their data and on the basis of these have tried to discern certain conventions according to which these were composed and are perceived.

An influential example of this kind of research is Kress and van Leeuwen's (2006 [1996]) Reading Images: The Grammar of Visual Design, which takes a social semiotic approach to the study of images. In their book Kress and van Leeuwen extend traditional linguistic notions of "literacy" and "grammar" from spoken word and written text to visual communication. Their aim is to study the underlying regularities according to which images are composed:

... despite the very large amount of work done on images, not much attention has been paid to the meanings of regularities in the way image elements are used - in short, to their grammar - at least not in explicit or systematic ways.... We intend to provide usable descriptions of major compositional structures which have become established as conventions in the course of the history of Western visual semiotics, and to analyze how they are used to produce meaning by contemporary image-makers. (Kress and van Leeuwen 2006 [1996]: 1) 
Kress and van Leeuwen analyze a variety of "text-objects" such as works of art, photographs, advertisements, websites, public signs, and newspapers. On the basis of these materials they demonstrate certain conventions at play in these objects, e.g., the different ways in which left and right (or top and bottom) are used to display information. Their book has stimulated a wide interest in multimodality (e.g., Mahin 2007; Kress 2010) and researchers have analyzed the visual properties of, among others, mathematical texts (O'Halloran 1999), children's picture books (Anstey and Bull 2000), animated films produced by children (Burn and Parker 2001), CD-ROMs of novels (Jewitt 2002), movie posters (Maiorani 2007), online newspapers (Knox 2007), and religious art (Haladewicz-Grzelak 2009). What all these studies have in common is that they base their analysis on products rather than the processes through which these products were assembled. Consequently, the design rationales of the original composers of the objects are not directly available to researchers, but have to be inferred.

A very different line of inquiry, video analysis of naturally occurring interaction (Goodwin 2001; Heath et al. 2010), has emerged out of ethnomethodology (Garfinkel 1967, 2002) and conversation analysis (Sacks 1992; Schegloff 2007) and has been explicitly concerned with the processes through which participants make their conduct recognizable ("accountable") to each other. This line of research is concerned with the in situ, ongoing production of sense and tries to exhibit the variety of practices through which participants display what they are doing to each other. Since the analysis is based on video recordings of activities, researchers can study directly the reasons for participants" conduct (where "reason" does not refer to some cognitive, "hidden" phenomenon, but to publicly displayed kinds of behavior).

Using video recordings thus not only allows the researcher to study the "doing" of various activities, but also to observe the mutual orientation of participants to each other's conduct. In that sense, it is not so much that the researcher analyzes particular forms of data, but rather uses the videotape to analyze how participants themselves construe each other's behavior. As Goodwin puts it:

... what the analyst seeks to do is not to provide his or her own gloss on how visual phenomena might be meaningful, but instead to demonstrate how the participants themselves not only actively orient to particular kinds of visual events (such as states of gaze), but use them as a constitutive feature of the activities they are engaged in (for example, by modifying their talk in terms of what they demonstrably see). (Goodwin 2001: 160)

Early investigations by Goodwin $(1981,1986)$ and Heath $(1982,1986)$ examined how participants orient to each other's gaze, gestures, and body posture in interaction. Subsequent studies explored, for example, how participants coordinate their activities in complex work settings (Heath and Luff 1992; Heath et al. 1995), 
the ways in which participants use multimodal resources in interaction (Mondada 2003, 2007), and how "professional vision" is organized in different occupations (Goodwin 1994).

The aim of this paper is to bring these two lines of research together. Thus the goal is not just to study the "visual grammar" (Kress and van Leeuwen 2006 [1996]), "visual logic" (Jayyusi 1988), or "visual language" (Horn 1998) of particular objects, but also to shift the emphasis from an analysis based on products towards an investigation of the processes through which such products are given their final shape. The challenge is to demonstrate how participants themselves design visual objects in such a way that these objects have the kinds of effects that have been previously analyzed.

The particular objects analyzed in this paper are storyboards that were produced by secondary school children as part of their engagement with Shakespeare's Macbeth (for an example see Figure 1). These are a perspicuous example for studying visual culture, since pupils have to transform a written text (Shakespeare's dialogue) into a multimodal storyboard, which consists of a series of pictures that nevertheless often contain text in the form of speech and thought bubbles.

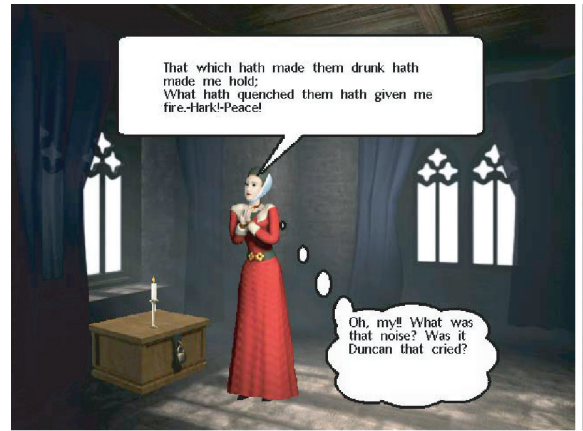

(a)

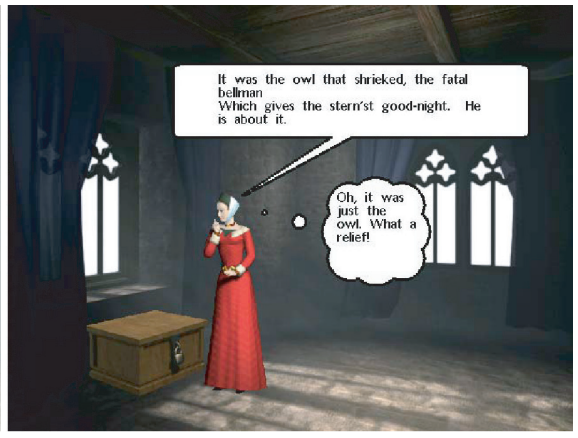

(b)

Fig. 1: The first two frames of a storyboard

The analysis will not, primarily, be based on the finished storyboards produced by students, but rather investigates video recordings of the students working on these storyboards. This allows us to investigate the processes through which these storyboards were constructed and to explore how the students themselves oriented to conventions during the composition of their storyboards.

There have been very few studies that have previously investigated the processes through which visual objects are assembled. This can be explained by the difficulties of both capturing and transcribing such activities. First, it is not 
straightforward to find situations in which participants explicitly topicalize their rationales for constructing a visual object in a particular way. For example, a video of a solitary designer working with a computer (and therefore saying very little) would be very difficult to analyze. Consequently, situations in which two or more participants collaborate (and talk to each other) are methodologically more promising. However, even in such situations it is not guaranteed that there will be many episodes in which the participants explicitly discuss the reasons for constructing a particular object. In order to assemble a variety of examples, many hours of video may be necessary. This was certainly the case for the data selected for this paper, which are episodes in which pupils explicitly discuss the placement of their speech bubbles. In more than ten hours of video recordings, only four instances were found.

Second, transcribing such episodes is very challenging. One of the advantages of basing the analysis only on products is that the researcher can easily make the data available to readers. However, this is not possible when working with video recordings where the researcher has to find ways of representing the video on paper. While relatively stable conventions have emerged for representing talk, transcribing the embodied conduct of participants (such as gaze, gestures, etc.) poses both theoretical and methodological challenges. As Ivarsson (2010: 176) puts it: "It is not without difficulty that the temporal and spatial arrangement of bodies, conduct and other events are transformed into the fundamentally spatial (non-temporal) arrangement better known as 'a page.’”

These difficulties are exacerbated in cases in which participants collaboratively work on a particular object, since the transcript must represent not only the talk and embodied conduct, but also the way that the object changes as a result of them. In this case, ways had to be found to display the storyboard frames that the pupils were manipulating on the computer screen. Since simple stills from the original video were not of a good enough quality to reproduce in a paper, the relevant storyboards were re-created and then linked in the transcript to the talk and embodied conduct.

Despite these challenges, there have been a few recent studies that have opened up the processes involved in assembling visual objects. For example, both Büscher (2005) and Murphy (2005) studied design meetings of architects, which allowed them, first, to investigate how the architects themselves interpreted their architectural drawings, and, secondly to study the design rationale behind the finished plans. Similarly, Ivarsson (2010) investigated design-ininteraction by observing the interactions of architecture students using a variety of software packages. Broth $(2008,2009)$ was able to video-record interactions in the control room during live television production, thereby exhibiting the conventions through which live television programs are produced (see also Engström 
et al. 2010). Finally, Laurier and Brown (2011) observed video editors in the process of working with video materials to investigate the decision-making involved in producing videos.

This paper follows these studies in examining the processes of designing particular objects, in this case, storyboards of Shakespeare's Macbeth. The analysis is based on episodes in which the pupils topicalized the placement of speech bubbles.

\section{The setting}

The study is based on video fragments taken from English lessons of a Year 9 class (pupils aged 13 to 14) studying Shakespeare's Macbeth (see Greiffenhagen 2008, 2012; Greiffenhagen and Watson 2009). Over the course of three months, the pupils spent the majority of their English lessons engaging with Macbeth through a variety of activities. The teaching approach taken was one that has been referred to as "active methods" (Gibson 1998; Franks 2003; Kress et al. 2005; Coles 2009), which consists of emphasizing the "dramatical” elements of Shakespeare by, for example, encouraging students to act out parts of plays or watching versions of the play (either on stage or on TV). In this case, the students were asked to use a new software tool called kar2ouche ${ }^{1}$ to construct in a series of frames their interpretation of scenes from Shakespeare's Macbeth. The software provides pupils with a library of pictures of illustrated backgrounds, characters, and props. Pupils create a series of frames by selecting appropriate backgrounds, characters, and props. Having placed the characters and props into the background, they then decide upon a pose for each character by selecting one of a range of alternatives depicting "anger," "worry," "resignation," "determination," etc. They can then attach speech and thought bubbles to the characters and insert into them any text they choose to type, including extracts from the play itself. Finally, they can write any notes they wish into a caption box at the bottom of each frame.

Having studied Macbeth for some weeks, working with the storyboarding tool became an additional activity for this class. Over the course of eight weeks, they had eleven lessons in the computer suite. All lessons in the computer suite as well as some in the "normal" classroom were recorded, one camera focused on a particular pair of pupils, the other alternatively on the teacher (during whole-class announcements) or on a second pair of pupils. It was thus possible to capture both what the teacher said to the whole class as well as the way individual pupil-

$1 \mathrm{http}: / /$ www.kar2ouche.com/ (for other computerized storyboarding tools see Madden et al., 2009). 
pairs worked with the software (and their occasional interaction with the teacher). The final storyboards of all sixteen pairs of pupils were also collected.

In the first session in the computer suite, the teacher gave the class the task "to produce a storyboard on the computer" of Act 2, Scene 2, which was the set piece for the national exams for this year (see Appendix 2). The task was to be completed over a number of lessons. Many students will have been familiar with storyboards, which, as Gibson (1998: 210) notes, "is a popular activity with students." What was new for these pupils was to produce storyboards using the computer. Consequently, the teacher spent most of the first lesson introducing pupils to the software. These instructions were quickly summarized at the beginning of the second lesson in the computer suite, ending with the summary in Transcript 1 , in which the teacher gives explicit instructions as to how students should visualize Shakespeare's text. ${ }^{2}$

\section{Transcript 1}

\begin{tabular}{|c|c|}
\hline I T: & think about how you're going to break up this opening >this opening part of this scene (.) how \\
\hline 2 & you're going to break it up (3.0) okay? (.) and start constructing your scenes (I.0) this is the \\
\hline 3 & work you're gonna save and work on for the next week $(2.0)$ so by this time next week $(I .0)$ \\
\hline 4 & we're gonna have a fair interpretation of this scene from each of you (I.0) any questions? (I.0) \\
\hline 5 & anybody not know what I want them to do? (I.0) so you're gonna put in your characters (.) put \\
\hline 6 & in your speech- >choose a bit of speech from the the text $($.$) and put in the thought bubbles ()$. \\
\hline 7 & and underneath say why you made the choices that you've made \\
\hline
\end{tabular}

In other words, students were asked to create a series of frames, choosing background and characters, using the speech bubbles (SB) for Shakespeare's text and the thought bubbles (TB) for their own understanding of the character's psychological motivation and thoughts; finally, the teacher wanted pupils to put their reasons for constructing the whole frame in this particular way in the caption box.

This paper focuses on how pupils dealt with the speech bubbles (rather than, say, how they chose backgrounds or poses for characters). Although the teacher's instructions were fairly explicit, they leave - as all instructions (cf. Sharrock and Watson 1985; Amerine and Bilmes 1988; Garfinkel 2002: ch. 6) - many aspects

2 I am following the conversation analytic transcript conventions developed by Gail Jefferson. The most important are the following: double parentheses [" $(())$ "] are used to mark transcriber's descriptions of events; single parentheses ["( )"] indicate uncertainty on the transcriber's part; underlined items ["item"] are audibly stressed; colons [“a::]" indicate prolongation of the immediately prior sound; the degree sign ["“"] is used as a softener; a dash ["-"] indicates a cut-off; an inbreath is denoted by a preceding circle [“o $\mathrm{h}$ "]; numbers in parentheses [e.g., "(0.3)"] denote a silence in tenth of seconds, while "(.)" denotes a micro pause of less than 0.2 seconds (see, for example, Atkinson and Heritage 1984: ix-xvi). 
underspecified and as tasks for the students to figure out for themselves. For example:

- How much of Shakespeare's text should they use in the speech bubbles? All of the text or just particular passages?

- How much of the text should they put in each frame? As much or as little as possible?

- Where should they place the speech and thought bubbles? Anywhere, or in specific places?

The video recordings of pupils working on the task allow us to investigate how both teacher and pupils found solutions to these questions.

\section{How much text?}

Act 2, Scene 2 starts with a long monologue by Lady Macbeth (see Appendix 2). When pupils began to work on the task, they were thus immediately faced with the question of whether they should put the entire monologue into their first frame. Transcript 2 provides an illustration of this. ${ }^{3}$

The pair of pupils (Bob and Sue) have already selected a background and provided Lady Macbeth with an empty speech bubble (SB). When Bob has marked and copied Lady Macbeth's entire monologue (line 1 in the Transcript; see Appendix 2, lines 2-9), Sue (who has control of the keyboard) inserts this into Lady Macbeth's speech bubble (line 3). It transpires that the current speech bubble is too small to "hold" all of the copied text, i.e., only shows some of the copied text (picture attached to line 3). Bob (who has control of the mouse) thus starts to resize the speech bubble (line 4). However, despite making the speech bubble extremely big (in fact, bigger than Lady Macbeth), it is still not big enough to hold all the copied text (picture attached to line 4). This prompts Sue to suggest to "just junk some," since "there's still loads to go" (lines 5-6), which can be taken as a suggestion to delete some of text in the speech bubble.

Sue's utterance is overheard by the teacher who approaches the pupils. The teacher suggests to the pupils to "break it [the monologue] into smaller pieces" and "have it over a couple of scenes [rather than just one]" (lines 8-9). The teacher further suggests that the pupils should have the character "moving around" (lines 11-12) while she is speaking, which is what the pupils subsequently do. Figure 2 displays their first two frames at the end of this lesson.

3 Images are connected with thin lines to the place in the transcript where they occur. The frames from the storyboards have been carefully re-created using the storyboarding software. 


\section{Transcript 2}

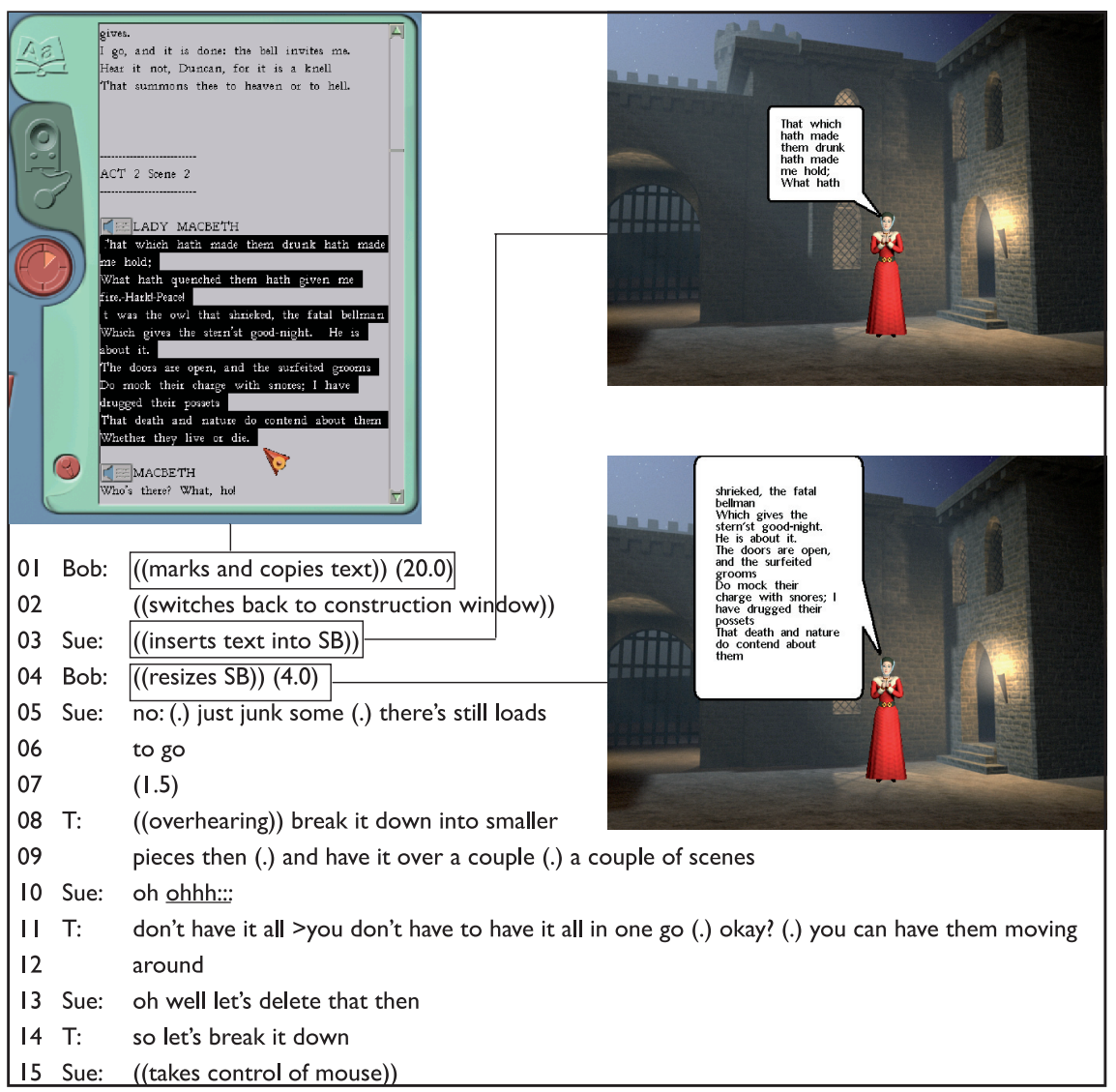

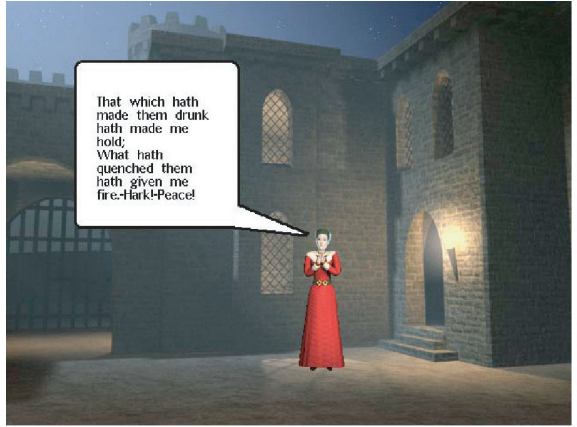

(a)

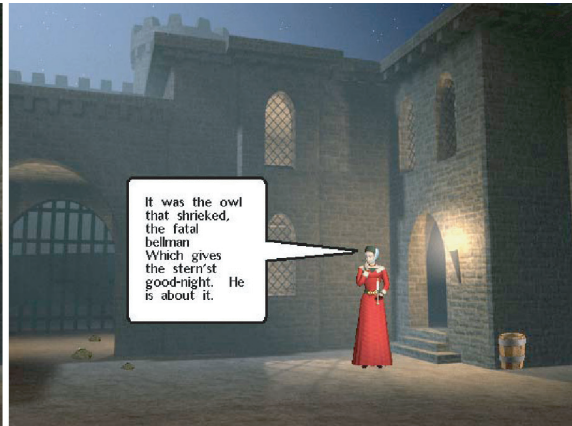

(b)

Fig. 2: The first two frames at the end of the lesson 
These two pupils have just started to work with the software and thus use the teacher's instruction to guide them. They begin by selecting a background and a character to start their first frame. They then proceed to "choose a bit of speech from the text" (teacher's instruction in Transcript 1, line 6). The text in the software is formatted to highlight the different speakers (in CAPITALS), but there is no formatting within a speaker's turn (see Appendix 2). It is therefore quite "natural" that the pupils initially select all of Lady Macbeth's turn.

However, when the teacher sees what they are doing, she is not happy with their "execution" of the task. There seem to be two reasons for this. First, in terms of the conventions of storyboards or comics, it is unusual to have a huge speech bubble dominating the whole image (the aim is to produce "an image with some text," not "a text with some image"). Second, the educational goal of the current task is not to put as much possible text in each frame, but rather to use this activity to reflect upon the text. Consequently, the teacher suggests that the pupils should split the dialogue over a couple of frames, which is what most other pupils did. Figure 3 gives another instance in which pupils have also split Lady Macbeth's monologue, but chosen slightly different shapes for her speech bubbles.

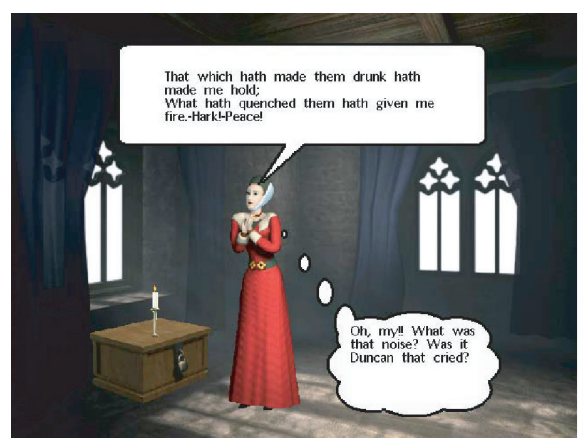

(a)

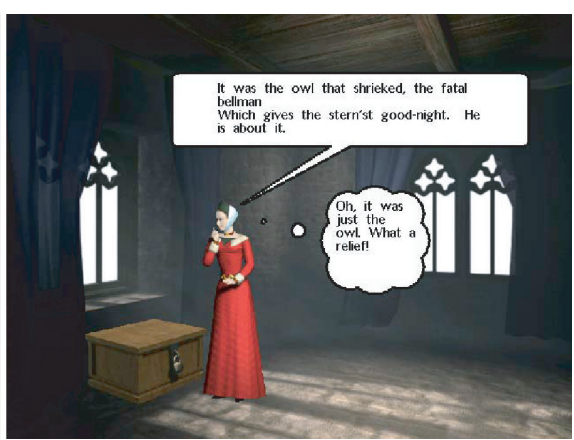

(b)

Fig. 3: The first two frames from another pupil-pair

Another aspect of the question of how much text pupils should use became a topic directly following the episode in Transcript 2. A pupil on the adjacent computer to Bob and Sue overheard what the teacher had told them and in turned formulated a question to the teacher (Transcript 3). 


\section{Transcript 3}

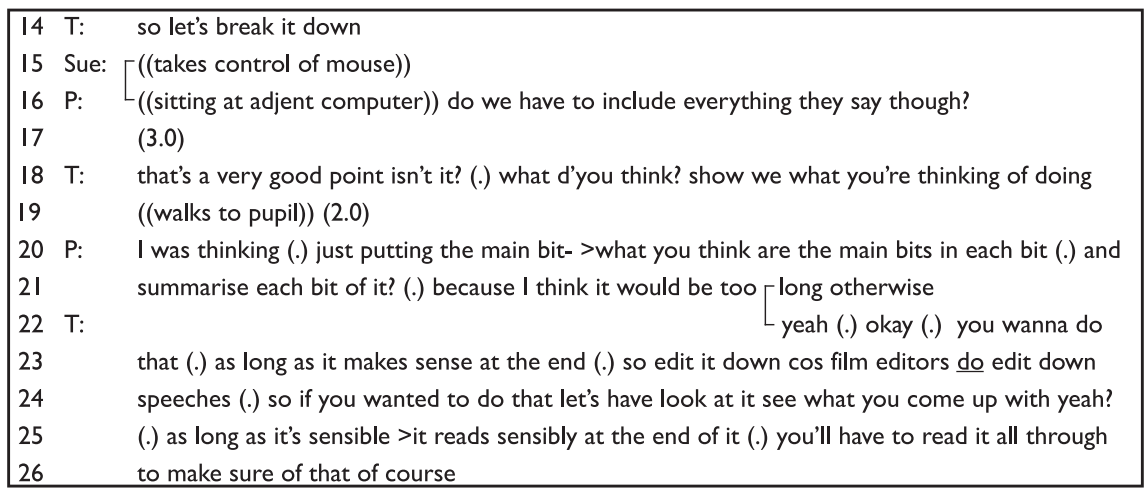

The pupil asks the teacher whether she has "to include everything they say" (line 16), which the teacher immediately characterizes as "a very good point" (line 18). The pupil elaborates that she was thinking of "just putting the main bit" of the text, in other words to "summarize" Shakespeare's text, because "it would be too long otherwise" (lines 20-21). The teacher accepts this suggestion (line 22) and also provides an account of why this would be acceptable: part of adapting a text for a film or theatre production is to edit it (line 23). So in that sense, what the pupil is proposing to do follows established practice in visualizing a text.

The question of how much text they should use did not create any serious difficulties for pupils when visualizing the text. If a character had a long turn, most of the pupils found a suitable place to break the utterance and continue it over one or two more frames. Others chose to cut the text. However, what did the pupils do when there was more one than character in the frame? After Lady Macbeth's initial monologue, Macbeth enters (see Appendix 2, line 12), presenting pupils with the questions of how many speech bubbles they should put in a frame (only one? one for each character? or more than two?).

\section{How many speech bubbles?}

As Yannicopoulou (2004) has shown, children are familiar with the role and function of speech bubbles, which they encounter in many forms:

Children's familiarity with speech bubbles, a somewhat arbitrary convention, since no one speaks or thinks with small clouds hovering above their heads, may be due to its omni- 
presence in the children's environments. Originating from comics, it has transferred to children's books, magazines, advertisements and environmental print. (Yannicopoulou 2004: 178)

Although students seemed to be familiar with the general function of speech bubbles, the teacher had given no clear guidelines as to how many speech bubbles should be in each frame. Looking at the final storyboards of the sixteen pairs of pupils, it quickly transpires that most of them decided to use only a small number of speech bubbles in each frame (see Table 1): only 10 out of 190 frames contained more than two speech bubbles.

\begin{tabular}{ll} 
Number of speech bubbles & Occurrence \\
\hline 0 & 19 \\
1 & 70 \\
2 & 91 \\
$>2$ & 10 \\
\hline
\end{tabular}

Table 1: Number of speech bubbles per frame

Since the teacher never explicitly instructed the class not to put more than two speech bubbles in one frame, this might point to a general preference for using only a small number of speech bubbles (perhaps an indication of an attempt not to overburden each image with too much text). However, there was one instance in which pupils inserted ten speech bubbles into one frame (see Figure 4). Furthermore, they decided to number these in order to indicate the order in which the speech bubbles should be read (the only instance where pupils did this).

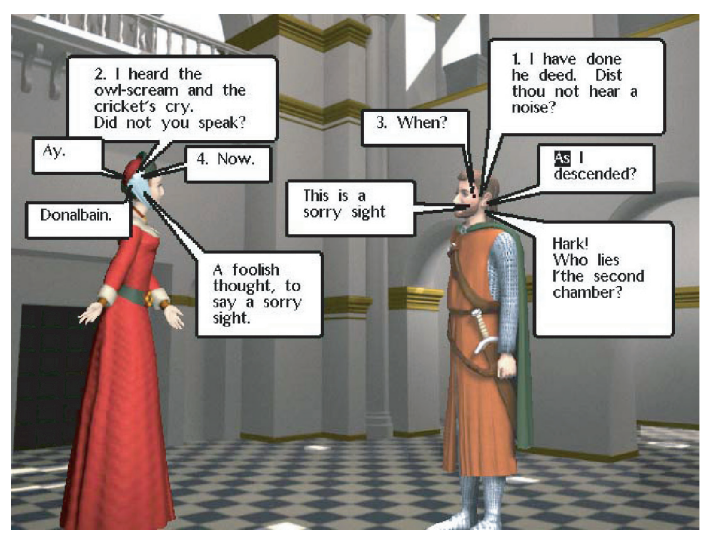

Fig. 4: Frame with ten partially numbered speech bubbles 
While the pupils are in the process of numbering their speech bubbles (they have numbered four of the speech bubbles and are in the process of numbering the fifth), the teacher appears behind them. Having observed what they were doing, she remarks that they have "too much" in their frame (Transcript 4).

\section{Transcript 4}

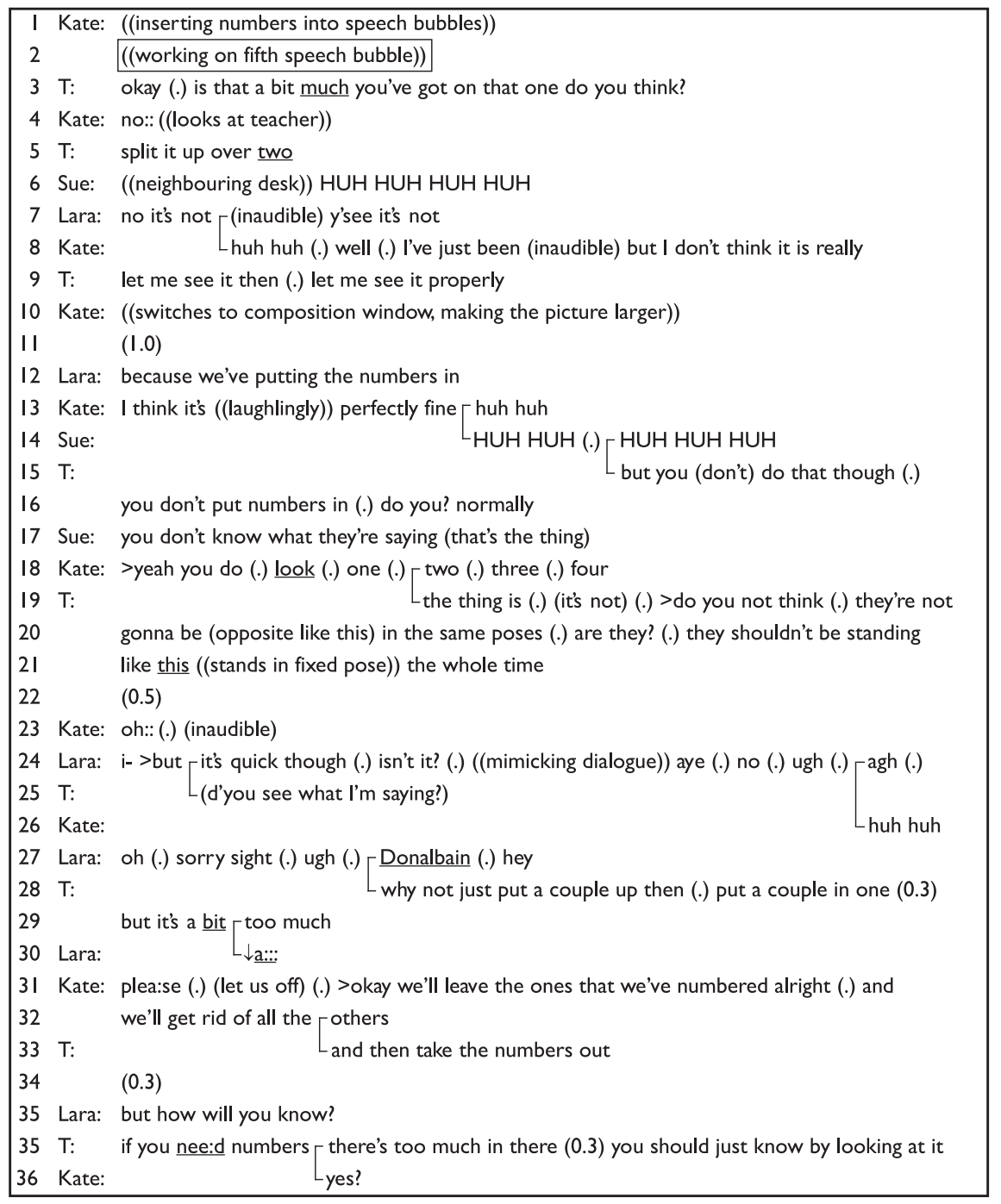

The teacher starts by commenting on what the pupils have done in the current frame: “is that a bit much you've got on that one do you think?" (line 3). This 
utterance, although having the form of a question, is audibly a reproach of their work (cf. Heritage 2002; Koshik 2002). In the classroom, formulations such as “don't you think that ..." or "might it not be better if ..." are a teacher's polite way of criticizing what pupils have done. Consequently, the teacher does not wait for a response from the pupils, but rather quickly continues with a suggestion as to how they could remedy this: "split it up over two" (line 5; compare Transcript 2, lines 8-9).

The pupils initially resist the teacher's characterization that there is "too much" (Kate in line 4, Lara in line 7) and provide a reason why they think it is acceptable to have so many speech bubbles: "because we've putting the numbers in," line 12). Kate also argues that "it's perfectly fine” (line 13), although her laughing intonation may suggest that this is not to be taken completely seriously. The teacher, in turn, takes up Lara's account and counters that "you don't put numbers in (.) do you? normally" (line 16). The teacher then gives an additional reason for having less dialogue in the current frame, namely, that the two characters should not be standing in the same position and pose during this long dialogue (lines 19-21). Kate seems to accept this (line 23), but Lara tries another line of defense: the way that they have used the speech bubbles depicts a "quick" dialogue (lines 24, 27).

At this point, the teacher repeats her earlier suggestion (to "split it over two") and furthermore explicates that they should "just put a couple in one" (line 28), since "it's a bit too much" (line 29). This is accepted by Kate who agrees to delete the speech bubbles that they have not yet numbered (lines 31-32). However, this is not accepted by the teacher as enough of a remedy. Not only should the pupils remove some of the speech bubbles, but they should also "take the numbers out" (line 33). This prompts Lara to ask: “but how will you know?” (line 35), which I take to mean: without the numbers in the speech bubbles how would you know in which order to read them? The teacher answers by saying that "you should just know by looking at it," and that therefore the fact that the pupils needed numbers shows that "there's too much in there" (line 35).

This fragment reveals at least two important aspects regarding the conventions underlying the visualization of Shakespeare's text.

First, the current frame is clearly unusual in having ten speech bubbles. It is not only the teacher who notices this: the whole episode is marked with laughter (e.g., by the pupil at the adjacent computer at lines 6 and 14; by Kate in lines 13 and 25, and by Lara's performance of a "quick" dialogue in lines 24 and 27). Why is the current frame so unusual and why does the teacher want the pupils to change it? This has much to do with the dominance of the text in the current frame. The whole point of constructing a storyboard is to visualize the text - not just to have one frame with a large number of speech bubbles. That is to say, there 
should be a balance between "visual" and "textual" elements - one that the current frame does not achieve. ${ }^{4}$

Second, the fragment touches upon the issue that will concern us for the rest of the paper, namely, the question of whether one can - or should - indicate the order in which the speech bubbles are to be read. These pupils, realizing that the larger numbers of speech bubbles makes it difficult for a reader to "follow" the dialogue, have come up with an innovative solution, namely, to number their speech bubbles. However, this numbering of speech bubbles goes against "normal” practice. As the teacher remarks: "you don't put numbers in (.) do you? normally" (line 16). Most comics and other artwork that include speech bubbles do not employ numbers to indicate the reading order, which seems to be known by the pupils in this class as well, since these two pupils were the only ones that used numbers.

Rather than using numbers to indicate the order, the teacher states that "you should just know by looking at it" (line 35). In other words, the teacher points to the existence of conventions (or a "grammar") that helps to indicate the order in which speech bubbles should be read. Lara's question ("but how will you know?," line 35) indicates that it is not clear to her how this might be done, i.e., that she might not be familiar with these conventions. In this respect, it is important to note that the teacher only indicates that there are conventions, but does not indicate what these are or how this could be done. The rest of the paper explores three instances where pupils do try to indicate to a reader an order of speech bubbles, not through the use of numbers but through the placement of speech bubbles within the frame.

\section{Where to place speech bubbles?}

\subsection{Reading paths}

Many authors have remarked that the placement of items within an overall image can structure how they will be perceived. Thus Kress (2003: 65) argues that placement has "meaning-effects," while Jewitt (2006: 45) states that the "place of an element in a composition affects the information value it has in the text." As an illustration, Kress and van Leeuwen (2006 [1996]: 179) show that within magazines

4 What the pupils may be trying to do is to orient towards the dialogue as a whole unit and therefore to preserve that unit in one single image, composed by "quick" turns. This orientation may explain their resistance to the teacher's critique. 
elements that are presented as given are placed on the left, while elements presented as new are placed on the right. The placement of items within an image can thus create a "reading path" (Kress 2003: 4), although such a reading path will be less "directive" than the one offered by "conventional" texts.

In densely printed pages of text, reading is linear and strictly coded. Such texts must be read the way they are designed to be read - from left to right and from top to bottom, line by line.... The pages we have described in this chapter are read differently - and can be read in more than one way. Their reading path is less strictly coded. (Kress and van Leeuwen, 2006 [1996]: 218)

When reading traditional texts, there is a very strong preference to read things first from left to right, and then from top to bottom (Morrison 1981: 247, n. 4; Watson 1997: 82). The same preference is used in the page layout of comics and graphic novels. McCloud (2006: 32) illustrates how a cartoonist should "guide [the] audience through [the] work from beginning to end" with the picture in Figure 5 .

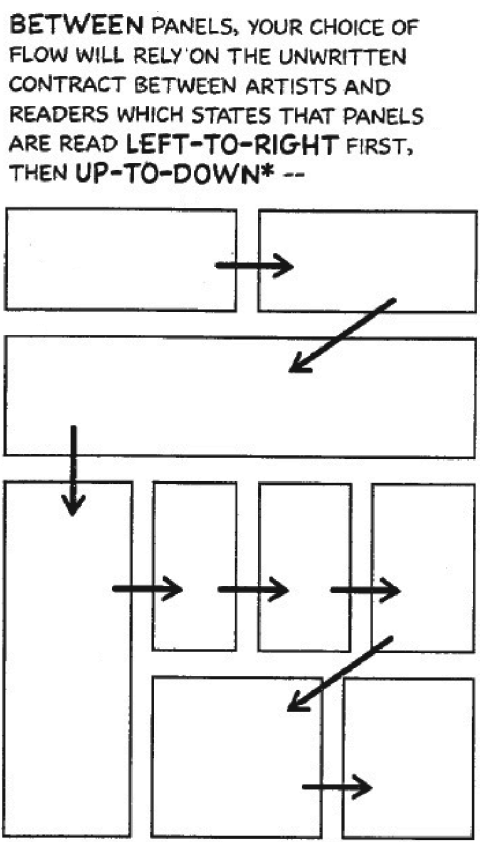

Fig. 5: "Reading path" in comics. Making Comics, copyright (c) 2006 by Scott McCloud. Reprinted by permission of HarperCollins Publishers

In other words, the placement of an item within the overall page can indicate the order in which it should be read (cf. Eisner (2008 [1985]: 42; Horn 1998: 135). 
As McCloud (1994: 7) puts it: "space does for comics what time does for film." Furthermore, these conventions for creating an explicit "reading path" are not just used for the reading paths between panels on one page, but also for speech bubbles within a panel. In other words, the placement of speech bubbles (or "balloons") depicts the sequence in which they should be read:

The arrangement of balloons which surround speech - their position in relation to each other, or to the action, or their position with respect to the speaker, contribute to the measurement of time. They are disciplinary in that they demand cooperation from the reader. A major requirement is that they be read in a prescribed sequence in order to know who speaks first. (Eisner 1990 [1985]: 26)

\section{STOPI Hils is rut Eack}

This manga collection is translated into English, but arranged in right-to-left reading format to maintain the artwork's visual orientation as originally drawn and published in Japan. If you've never read comics this way before, take a look at the diagram below to give yourself an idea of how to go about it. Basically, you'll be starting in the upper right-hand corner, and will read each word balloon and panel moving right-to-left. It may take a little getting used to, but you should get the hang of it very quickly. Have fun!

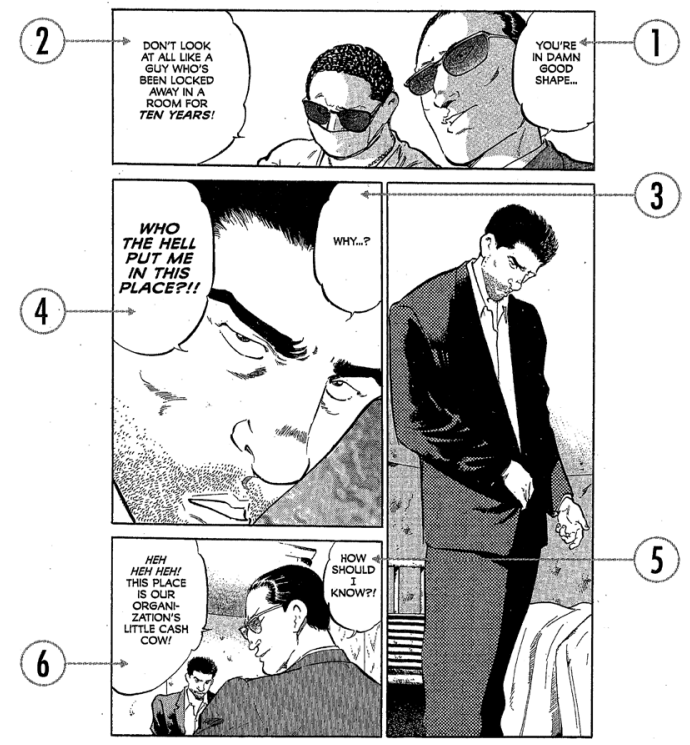

Fig. 6: Reading instruction for a Japanese manga ${ }^{5}$

5 Old Boy, copyright by Garon Tsuchiya and Nobuaki Minegishi. Originally published in Japan by Futabasha Publishers. Reprinted with permission of Dark Horse Comics, Inc. and Futabasha Publishers. 
This is the reason why most comics do not use numbers (as the two girls did in the previous fragment): they are designed in such a way that a reader can easily find the order in which the speech bubbles should be read, making use of the preference of reading from left-to-right and top-to-bottom. This order is, of course, highly conventional and specific to Western cultures. In Japanese manga, for example, a different order is at play. Japanese books start at what is in the Western context the "back." In cases of cross-cultural contact (as in the case of translating a manga from Japanese to English, but leaving the original artwork intact) an explicit instruction for Western readers may therefore be necessary. Figure 6 is the "first" page in an English translation of a Japanese manga.

This page instructs the reader not only that the first page of this book is what would be the last page in an English book (and the book has therefore to be read "from back to front"), but also that within each page the Western conventions of reading are not at play. Rather than reading from left-to-right, both panels and speech bubbles should be read from right-to-left, although the top-to-bottom order is left intact. ${ }^{6}$

\subsection{Above/next to character}

Given these considerations, the question arises: How did pupils decide where to place their speech bubbles? To which the initial answer has to be: for the most part, they did not "decide" at all. That is to say, there were very few occasions in which pupils explicitly discussed the placement of speech bubbles. They simply did not topicalize this question. When looking at the videotapes, the placement of speech bubbles was typically just "done," but rarely "discussed.” The absence of any explicit discussion among pupils might indicate that there were very strong shared conventions governing the placement of speech bubbles. Looking through the final storyboards only one clear preference with respect to the placement of speech bubbles emerges: speech bubbles were placed above/next to the charac-

\footnotetext{
6 This seems to reaffirm Goody's (1977: 130) observation that left-to-right is more conventional (i.e., more variable across cultures) than top-to-bottom: "But left to right is a more "conventional" movement than that from top to bottom. Indeed, systems of writing that begin at the right and move in the opposite direction, such as Arabic and Hebrew, clearly have a different order of priority. No-one, I think, has employed a system of writing that starts at the foot of the page and moves to the top, then jumps to the bottom again. The vertical hierarchy is more compelling, more insistent, than horizontal differentiation; it is the difference between head and foot in one context and right and left on the other."
} 
ter who is "speaking" them. In other words, it was the position of the character that determined the position of the speech bubble (and the imperative for the bubble does not obscure the figure, so "above the head" is kind of empty space, narratively speaking). This has interesting consequences for any potential "reading path," i.e., the order in which the speech bubbles might be read. In contrast to the conventional comic rules (discussed by McCloud above), the top left speech bubble is not necessarily the first in the dialogue depicted in a particular frame. This can be illustrated by looking at how four different pupil-pairs visualized lines 24 and 27/8 from the play (see Appendix 2). Two of them put Macbeth (and therefore also Macbeth's speech bubble) on the left (Figure 7), while the other two put them on the right (Figure 8).

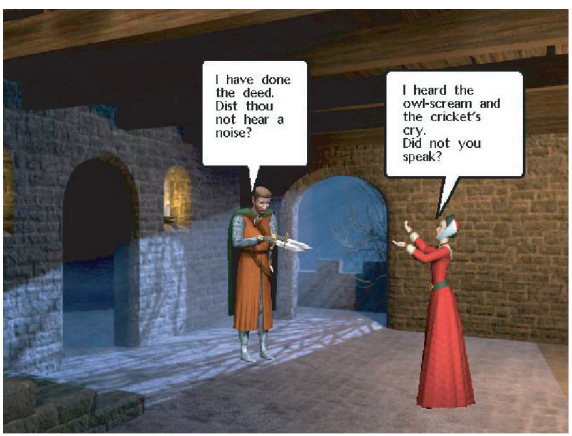

(a)

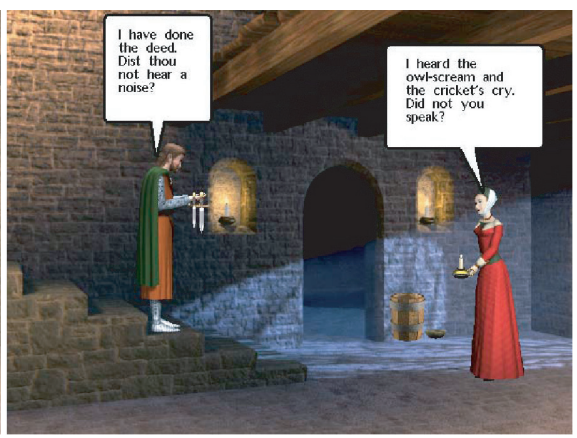

(b)

Fig. 7: Macbeth (and his speech bubble) on the left

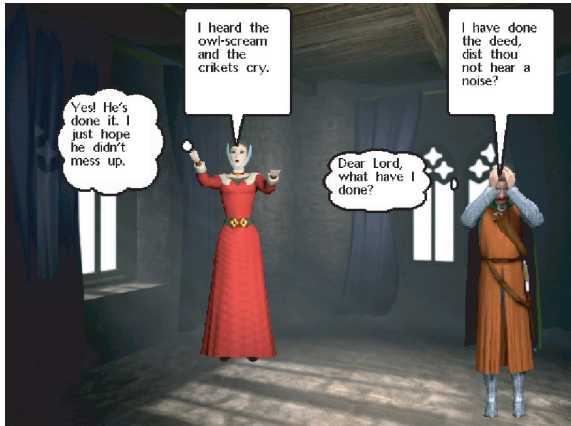

(a)

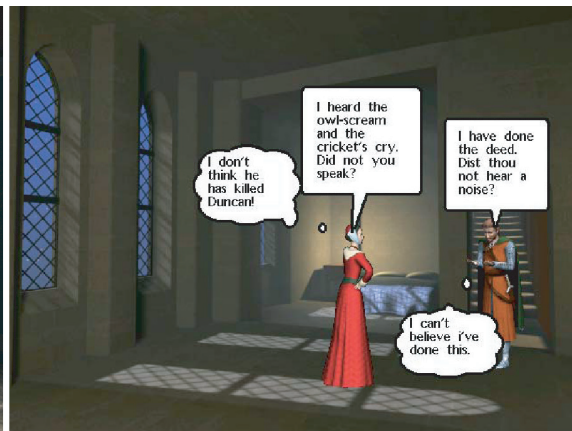

(b)

Fig. 8: Macbeth (and his speech bubble) on the right 
In other words, it seems that for many pupils it was the position of the character that determined where a speech bubble was placed, rather than the attempt to create a "reading path." This can be further illustrated in the following episode (Transcripts 5.1 and 5.2), in which one pupil wants to move Macbeth from the right to the left side, since it is Macbeth (rather than Lady Macbeth) who speaks first.

\section{Transcript 5.1}

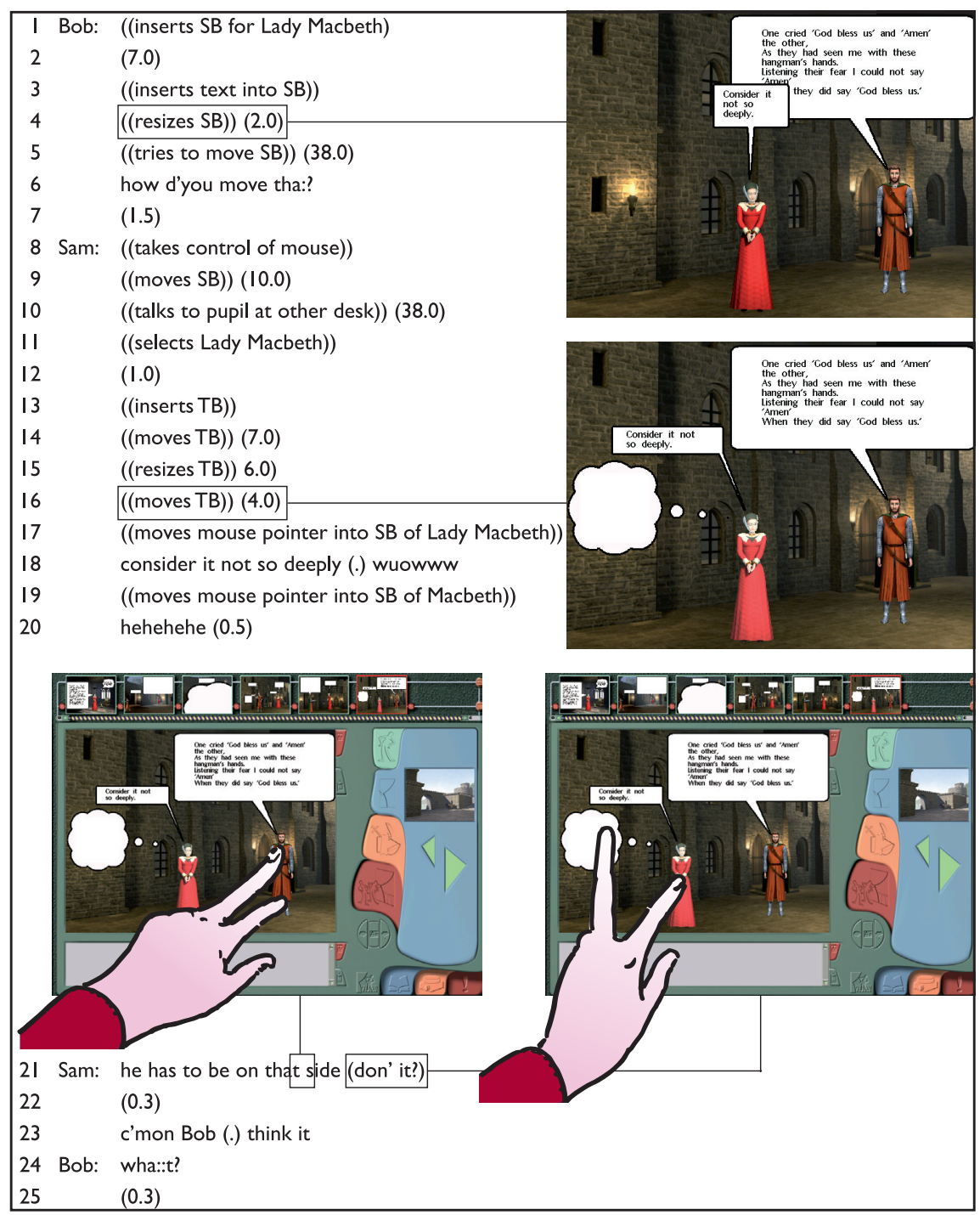


In this episode, the two pupils are working on their sixth frame. Bob, sitting on the right and currently in control of the mouse, has already inserted a speech bubble and the text for Macbeth and is now in the process of doing the same for Lady Macbeth. He inserts a speech bubble (line 1), copies the corresponding text (line 3), before resizing the speech bubble (line 4), so it nicely "wraps around" the text (in this case this means making the speech bubble smaller).

Since the speech bubble of Lady Macbeth overlaps with that of Macbeth (see image attached to line 4), Bob tries to move Lady Macbeth's speech bubble (line 5). However, he is not able to do so (with this prototype of the software it is relatively difficult to find the exact spot on which to click so that one can move, rather than resize, a speech bubble). Bob asks Sam how one can move a speech bubble (line 6), which prompts Sam to take control of the mouse (line 8) and to move Lady Macbeth's speech bubble (line 9).

Sam then proceeds to the next "step" in the teacher's task: after inserting the speech bubbles, they should insert thought bubbles in which they type their interpretation of the character's motivation. Sam inserts and positions a thought bubble for Lady Macbeth (lines 13-16). Having done so, he moves the mouse pointer (MP) into the speech bubble of Lady Macbeth (line 17), reading it out aloud (line 18). He does the same with Macbeth's speech bubble (line 19), but then releases the mouse and remarks that "he [Macbeth] has to be on that side [at the position of Lady Macbeth]" (line 21), using his left hand to point to what "he" and "this side" refer to (see the drawings attached to line 21, occurring at "that side" and "don't it" respectively).

Bob's non-response (line 22) prompts Sam to try to get Bob’s attention. Sam turns to Bob and states "c'mon Bob (.) think it" (line 23). However, Bob does not seem to see what Sam wants him to do or pay attention to (line 24), so Sam repeats his remark (Transcript 5.2).

Sam repeats his observation from line 21 in line 26: "he's [pointing to Macbeth] saying that first [pointing to the speech bubble of Lady Macbeth]." Bob's reply, “yeah:?" (line 28) seems to accept this, but the questioning intonation further suggests that Bob does not see what implications Sam's observation might have. In other words: So what if the text in Macbeth's speech bubble comes before the text in Lady Macbeth's speech bubble?

Rather than explaining himself "in words," Sam demonstrates what he is thinking by directly changing the current frame. Prefacing his actions with "let's think of it like this" (line 31), Sam starts to re-arrange the elements in the frame: he moves the speech bubble of Macbeth and then Macbeth himself to the left (lines 30, 32), before doing the same for Lady Macbeth and her speech bubble (lines 34, 35). He then also moves the thought bubble (line 38) and "fine-tunes" the elements, before moving the mouse pointer into Lady Macbeth's speech 


\section{Transcript 5.2}

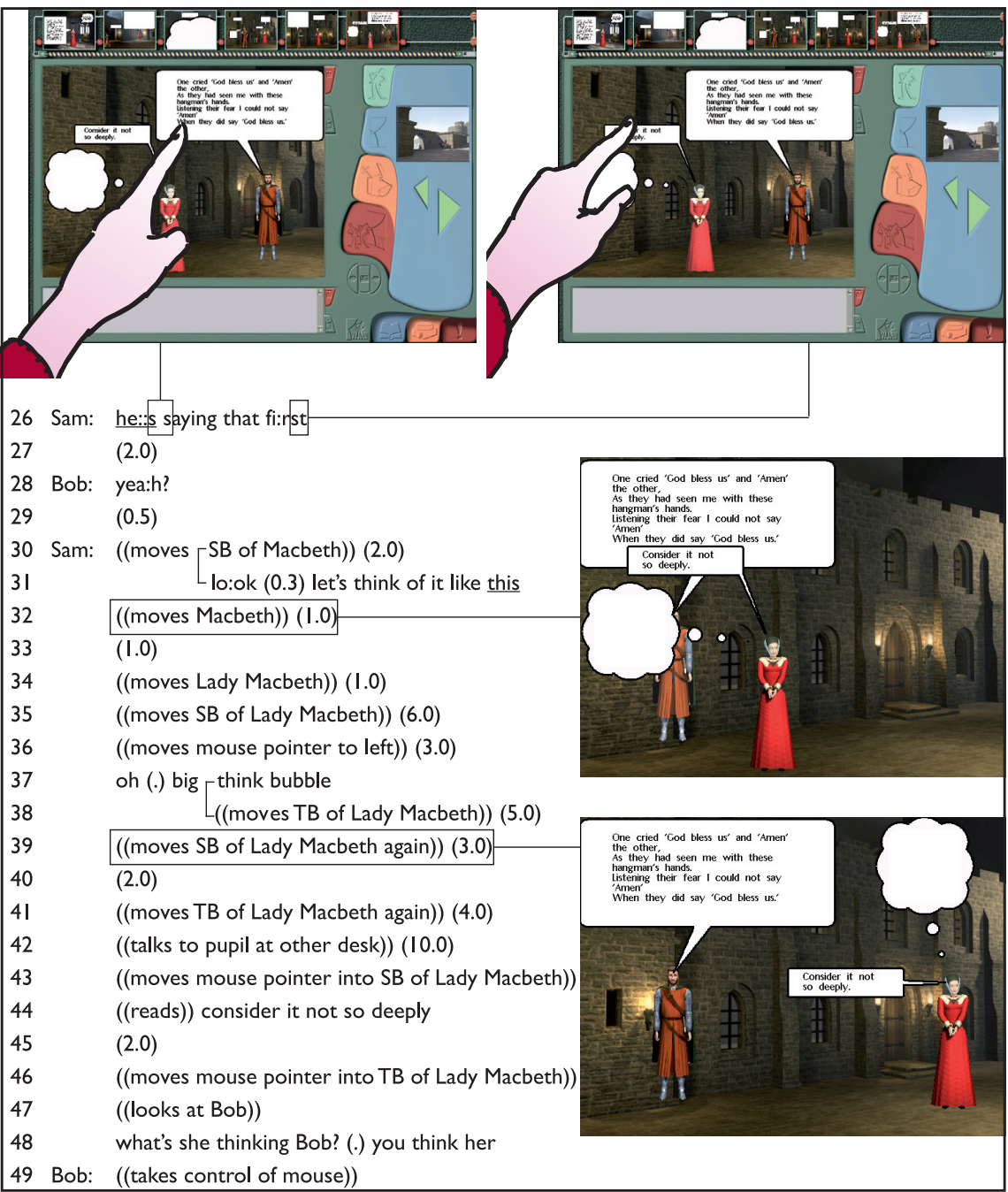

bubble, reading it aloud (lines 43-44). He finishes by moving the mouse pointer into Lady Macbeth's thought bubble, looking at Bob and prompting him to start working on the thought bubble: "what's she thinking Bob? (.) you think her" (line 48).

This fragment is one of only three instances, in which pupils explicitly discuss the placement of the speech bubbles in a frame. When preparing to work on the thought bubbles, Sam notices that it is actually Macbeth who speaks first in 
the current frame (line 26). For Sam this seems to imply that Macbeth should therefore be on the left (line 21, picture attached to line 39). In other words, Sam orients to the conventional left-to-right "reading order" of speech bubbles: as the frame stands, it is Lady Macbeth's speech bubble that is read first (which is in contrast to the "actual" reading order from the play).

Sam's solution also demonstrates his orientation to the aforementioned rule that it is the position of the characters that determines the position of the speech bubbles. Sam does not just move Macbeth's speech bubble, but rather changes the position of both Macbeth and the speech bubble, which are treated by him as a unit. That is to say, an alternative solution would have been to only change the position of the speech bubbles. This is something that another pupil-pair did for another part of the dialogue (Figure 9).

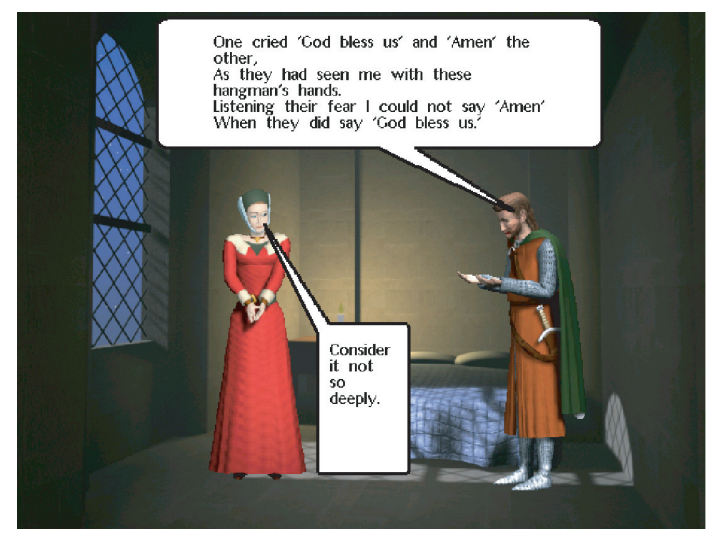

Fig. 9: An explicit reading path

In that instance, although Macbeth is standing on the right, the pupils were able to indicate that it is Macbeth who speaks first, since they placed the speech bubble of Lady Macbeth (who is standing on the left) below that of Macbeth. However, such a solution was extremely rare (only two instances among the roughly two hundred frames of the final storyboards). In general, pupils adhered to the speech bubble above/next to character convention. On the one hand, this might have been a result of the affordance of the software itself: when inserting a speech bubble, the speech bubble would appear above the character's head. On the other hand, it might be that pupils were familiar with the conventions for speech bubbles in some online games, where speech bubbles would appear above characters regardless of the order in which characters "spoke" (Figure 10). 


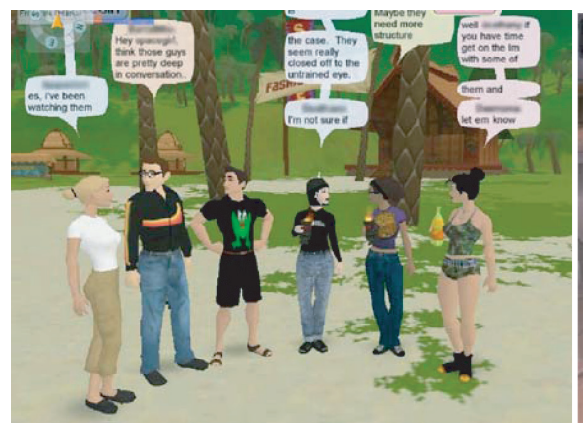

(a)

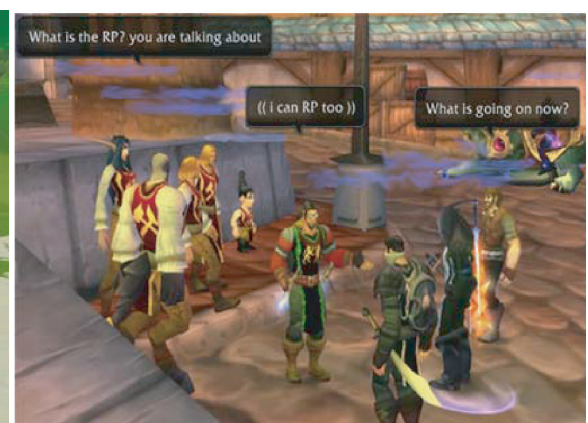

(b)

Fig. 10: Two screenshots from online games ${ }^{7}$

This fragment also sheds light on an aspect that will reoccur: it was only one of the two pupils (Sam) who was concerned with the question of where Macbeth's speech bubble should be placed. The other pupil (Bob) remained non-involved throughout this episode. In fact, Bob's puzzled reaction to Sam's observation (line 28) might point to the fact that although Sam, on this occasion, tries to depict an explicit reading order for the speech bubbles, Bob does not treat this as particularly important, since he in no way comments on Sam's eventual solution (there is no "yeah, good point" or something similar). It might be that Bob is orienting to the size of the bubble, which could be seen to solve the problem of order, since Macbeth's bubble is bigger than Lady Macbeth's bubble (unfortunately, Bob is not very responsive so it is difficult know what he is orienting to).

\subsection{Down the middle}

As already mentioned, the majority of frames only contained one or two speech bubbles and it was the position of the character that determined the position of the speech bubble. In contrast, in the next episode, the pupils inserted six speech bubbles in one frame and found a way of indicating a clear reading order, which did not involve numbering the speech bubbles (in the way that the two girls did in Transcript 4). In this episode, the two pupils have just started to work on the third frame. Having inserted the two characters, Macbeth and Lady Macbeth, one

7 Grateful acknowledgement is made to Barry Brown and Ulrika Bennerstedt for permission to use these images: see Brown and Bell (2004: 352) and Bennerstedt (2008: 73). 
pupil suggests where they should be placed in order to create "room" for the eventual speech bubbles.

\section{Transcript 6.1}

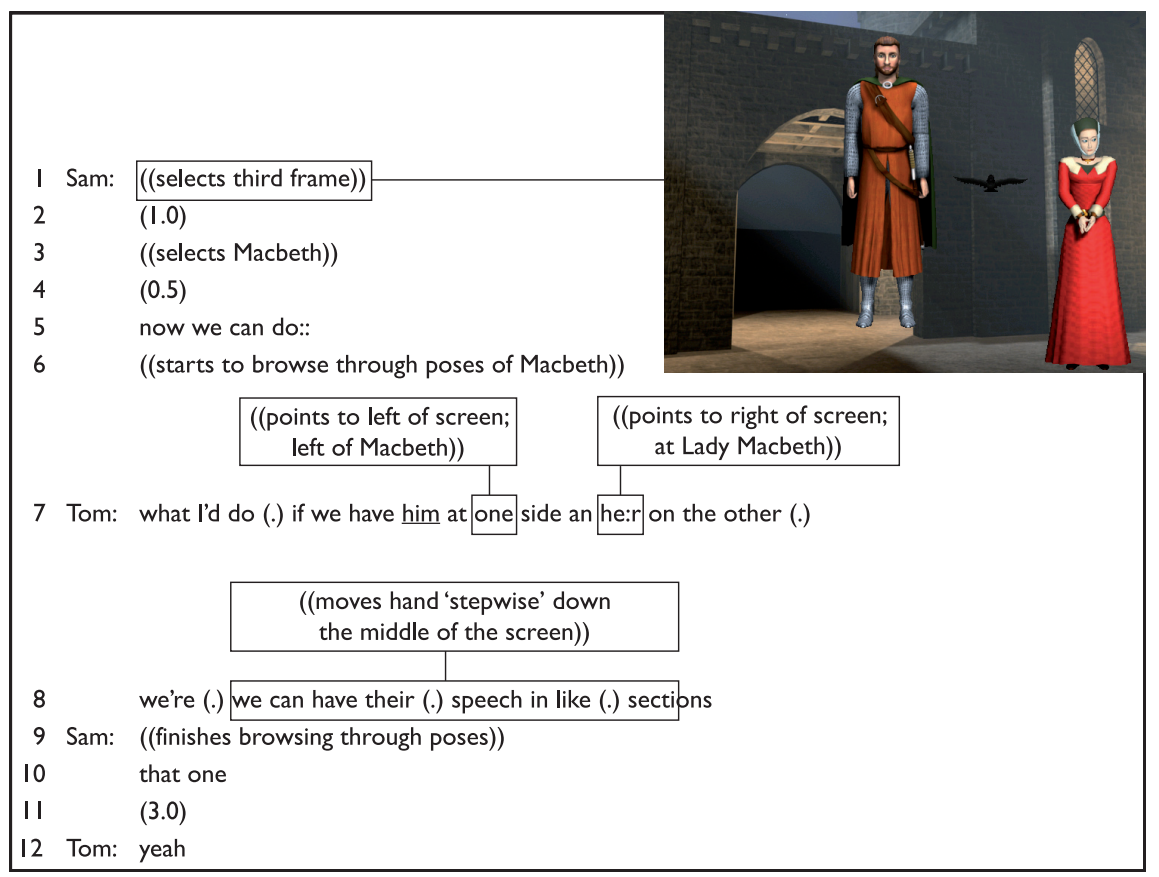

The two pupils have just finishing correcting some missing elements in their second frame and now return to continue working on their third frame (line 1). Sam, sitting on the right and currently in control of the mouse, selects Macbeth (line 3) and starts to search for a suitable pose for him (line 6). At this point, Tom, sitting on the left, points to the screen and suggests that they could "have him [Macbeth] on one side [on left] and her [Lady Macbeth] on the other [on the right]" (line 7). He then elaborates that they could then "have their speech in like sections" (line 8), moving his hand down the middle of the screen in the space in between the suggested new positions of Macbeth and Lady Macbeth.

Tom seems to be concerned with the placement of the still-to-be-inserted speech bubbles. He wants to move Macbeth to the left and Lady Macbeth to the right, using the space in between them for "their speech," which would be "in sections" (indicating, possibly, that he plans to have more than two speech bubbles in this frame). Sam, though, does not react to Tom's suggestion. Instead, Sam 
continues to browse through different poses and finally finds a pose for Macbeth that he likes (lines 9 and 10). Tom, after a short pause (during which he is possibly waiting for Sam to react to his suggestion), agrees to the suggested pose (line 12).

\section{Transcript 6.2}

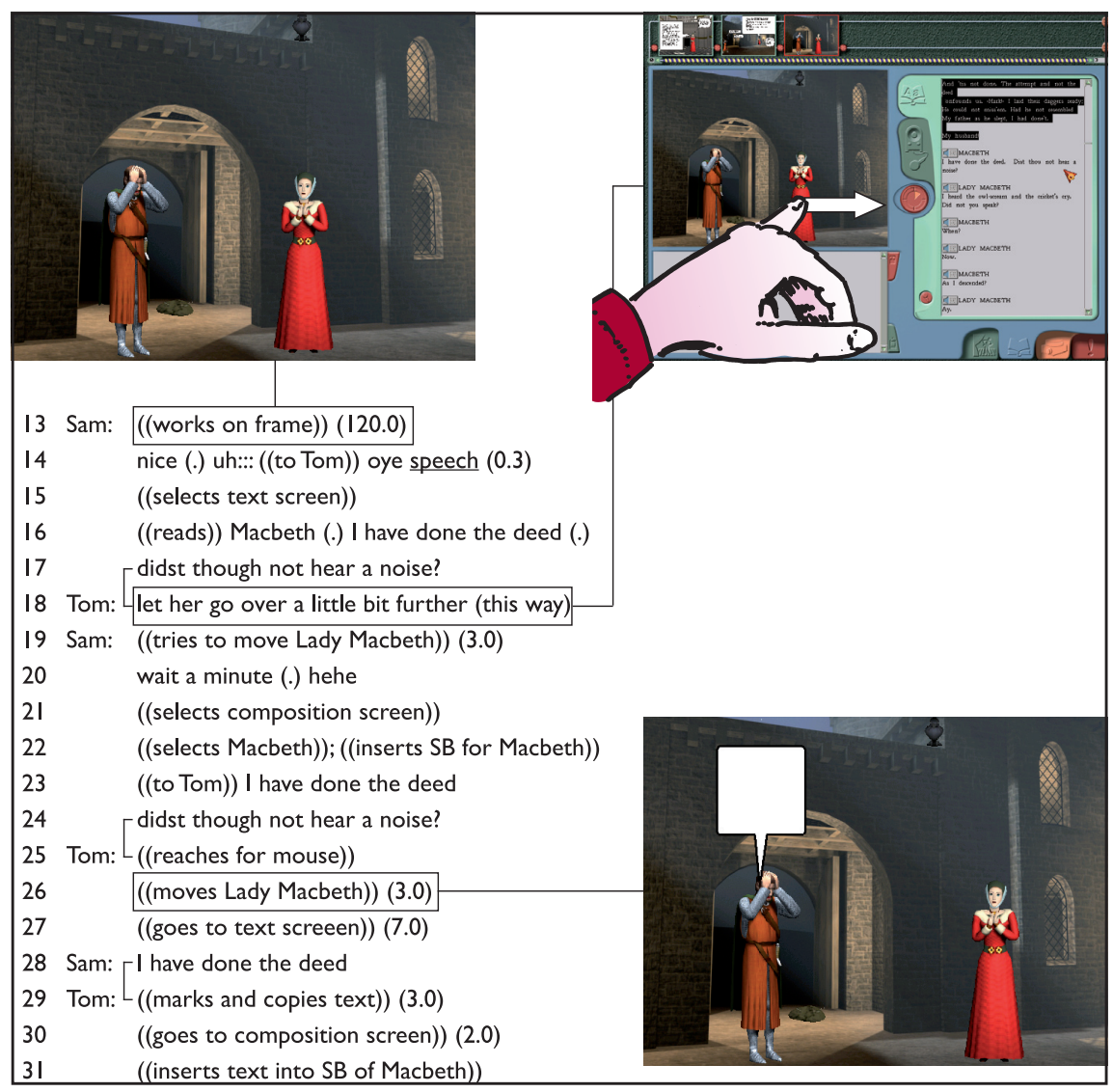

Sam now proceeds to work on the frame, changing the position and size of both Macbeth and Lady Macbeth (see image attached to line 13). He then turns to Tom and prompts him to move on to the next step in the task, namely, "speech" (line 14) bubbles. Sam changes from the composition screen to the text screen (in order to get access to the text from the play) and reads out the next line from the play (lines 16/17; see Appendix 2, lines 23/24).

At this moment, Tom points to Lady Macbeth and asks Sam to "let her go over a little bit further (this way)" (line 18), moving his hand to the right (indicating 
the direction in which he would Lady Macbeth to be moved). Sam tries to move Lady Macbeth (line 19), but does not succeed (since it is not possible to move a character in the text screen). Sam then changes back to the composition screen (line 21), inserts a speech bubble for Macbeth (line 22), and then instructs Tom which text to type in the speech bubble (lines 23/24). In other words, Tom tries to pursue his earlier suggestion of creating a space in between Macbeth and Lady Macbeth in which they could have "their speech in like sections" (line 8) and Sam tries to comply (line 19), but when this does not work he quickly abandons Tom's proposal. Yet as soon as Tom takes control of the mouse (line 25), he moves Lady Macbeth to the right (line 26). Only then does he go back to the text screen and copies the text for Macbeth (lines 27-31).

\section{Transcript 6.3}

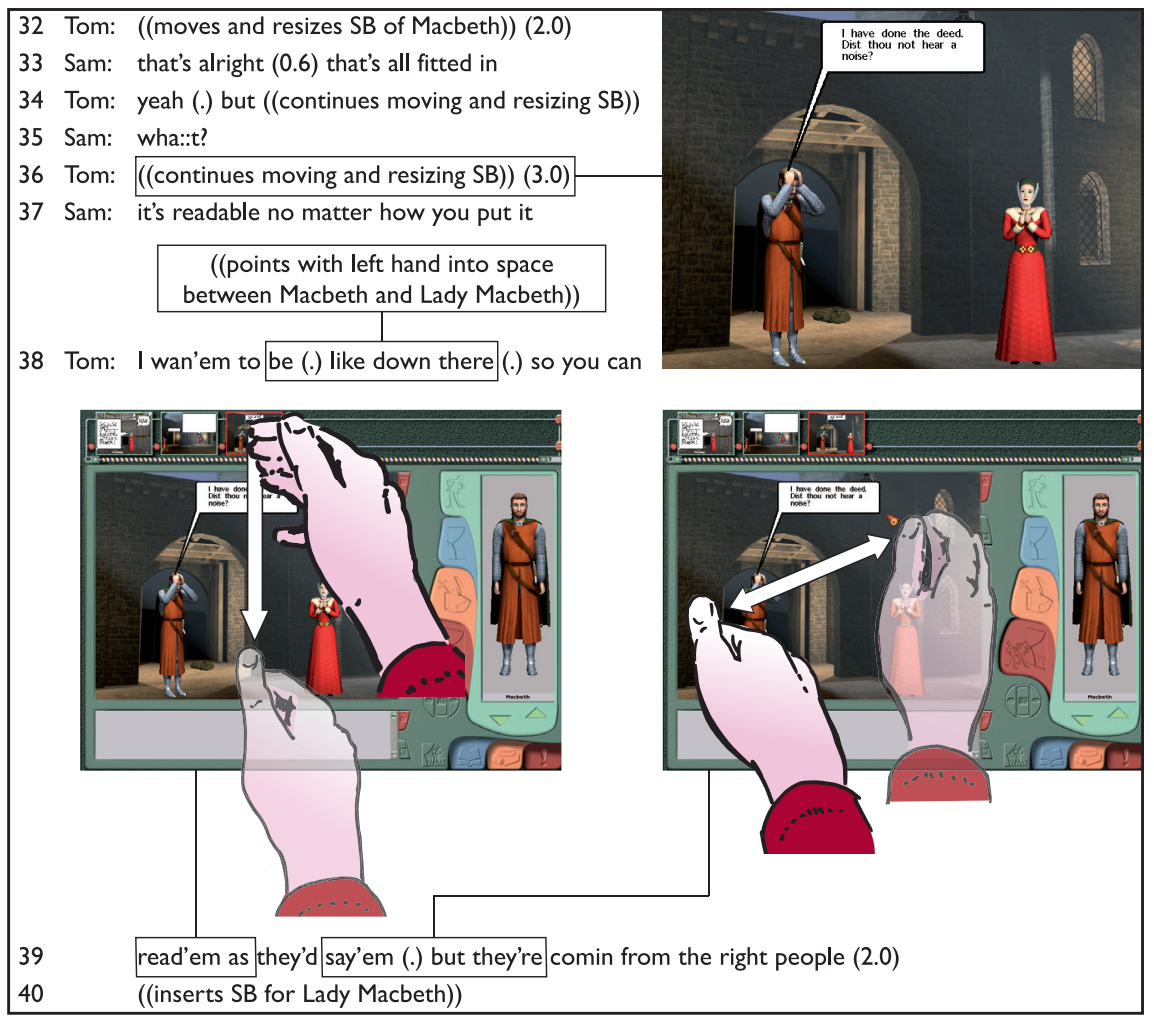

Having inserted the text into Macbeth's speech bubble, Tom starts to resize and reposition the speech bubble (line 32). This takes rather longer than usual, which 
prompts Sam to say: “that's alright (0.6) that's all fitted in” (line 33). In other words, Sam seems to wonder what more needs to be done once the speech bubble has been resized so it displays all the inserted text (in contrast to Transcript 2, line 4, the speech bubble here does display all of the inserted text). Tom, however, continues to resize and reposition the speech bubble, uttering "yeah (.) but" (line 34), indicating that there still is work to be done. Sam's "wha::t?” (line 35) displays that for Sam there is no "but," i.e., that Tom should move on to the next "step" (in this case, Lady Macbeth's speech bubble), since the current speech bubble is "readable no matter how you put it" (line 37).

At this point, Tom seems to finally have found a position for the speech bubble that he likes. He lets go off the mouse and explains to Sam what he is trying to do: "I wan'em [the speech bubbles] to be (.) like down there [in the space between Macbeth and Lady Macbeth] (.) so you can read'em [moving his hand from to top to bottom] as they'd say they'em (.) but they're [moving his hand back and forth from Macbeth to Lady Macbeth] coming from the right people” (lines 38-39). In other words, Tom is thinking of placing a number of speech bubbles in the middle of the screen, between Macbeth and Lady Macbeth, so that they can be read in sequence (from top-to-bottom), but are still connected to the person who is saying them. This is exactly what he subsequently does, as the frame at the end of the lesson shows (Figure 11).

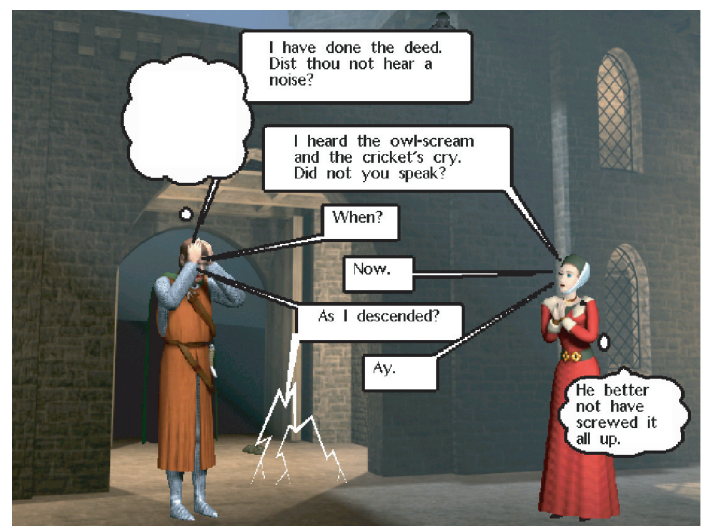

Fig. 11: Frame at the end of the lesson

What is noteworthy in this episode is that Tom very early devises a plan for the placement of the speech bubble in the frame. Retrospectively, we can see what his early utterance to "have their speech in like sections" (line 8) was meant to suggest - and why he was so insistent to have Macbeth on the left and Lady Macbeth 
on the right (lines 7, 18, and 26). Tom uses a variety of gestures over the current frame to indicate how the frame should look (lines 7 and 8, line 18, and lines 38 and 39).

This is clearly an innovative way of placing speech bubbles and creating a "reading path" for the speech bubbles. Comparing Tom's placement of the speech bubbles in Figure 12 with the speech bubbles in Figure 4, we can see that the former does suggest a clear reading path while the latter does not. In other words, Tom finds a solution so that a reader sees the order in which the speech bubbles should be read "just ... by looking at it" (teacher in Transcript 4, line 35).

Tom's solution not only indicates a clear reading path, it also manages to display another aspect of the dialogue that the girls in Transcript 4 were trying to convey: namely, a "quick” dialogue. What is only barely captured in the transcript (line 39, image connected to "say'em"), but clearly visible in the video, is how Bob visualizes the "speech in sections" as a back-and-forth dialogue between Macbeth and Lady Macbeth (his hand moves rapidly from one to the other, a bit like a ping pong ball).

Finally, as in the previous episode, only one of the pupils is explicitly concerned with the placement of the speech bubbles. While Tom in this episode clearly orients to the placement of the speech bubbles and the resulting "reading path," it is noticeable that Sam does not orient to this. Sam does not "disagree" with what Tom is doing (although he shows some impatience), but he does not seem to care. There is no visible reaction to Tom's suggestion and explication of what he is trying to do.

\subsection{Ambiguity in reading path}

The previous two fragments were instances in which one pupil wanted to place speech bubbles in particular way to indicate a reading path. In this final fragment we encounter a situation where the pupils have difficulty finding the reading path in a frame that they have previously created.

The episode occurs at the beginning of the fifth lesson in the computer suite, after the pupils have loaded their work from the previous lesson. To proceed with their task the pupils need to establish which is the last line from Shakespeare's that they have used (i.e., put in a speech bubble). They therefore look at the last frame that they have worked on (Figure 12). In order to find the last line that that they have used, they try to read the dialogue visualized in the frame from beginning to end. This turns out to be difficult, since it is not clear which of the two speech bubbles of Macbeth should be read first: the one "most on the left" or the 


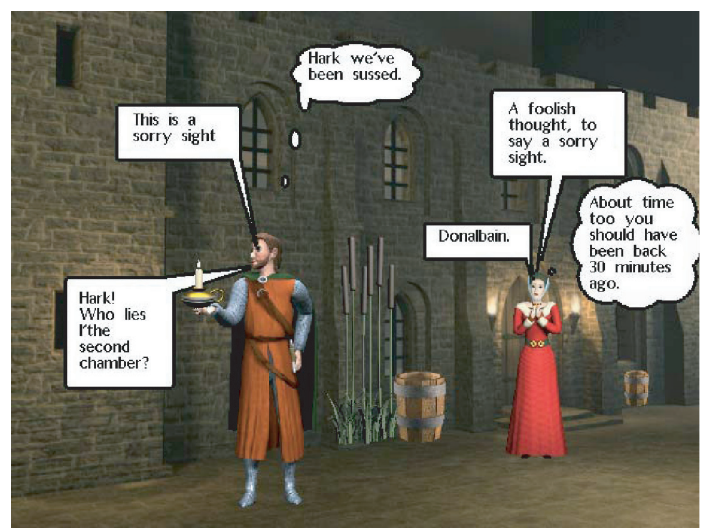

Fig. 12: An ambiguous reading path

one "most on top." Put differently, in this frame it is not immediately clear which of the two conventional rules, top-down and left-right, should dominate (compare with Figure 11, where only one of these two rules, namely, top-down, is in play).

In order to know which text they should put in the fifth frame, Sam goes to back to the fourth frame to see "where we are" (Transcript 7.1; line 6) in the text. An obvious way of finding "where we are" is to locate the first speech bubble in the frame and then read the remaining speech bubbles in sequence, thereby ending with the last line from the play that has been visualized. Sam tries to do just that: he moves the mouse pointer "searchingly" around the screen (line 7), finally settling on the left bottom speech bubble (line 8), which he reads aloud: "hark (.) who lies" (line 9).

He stops (line 10) and moves the mouse pointer instead into the top left speech bubble, reading that aloud: "this is a sorry sight" (line 11). He then does the same for the top right speech bubble (line 12) and bottom left speech bubble (line 13). At this point Bob interrupts Sam, suggesting that "that [the bottom left speech bubble] should be up there [at the position of the currently top left speech bubble] cos that [in the bottom left speech bubble] says it first" (line 15). Using his hands, Bob indicates that he wants to swap the two speech bubbles of Macbeth, putting the one in the left corner on top, since it is that one that is read first. Bob, in other words, does two things: he indicates that Sam has currently identified the wrong speech bubble as the "first" (namely, the top one); and he furthermore wants to place that the speech bubble identified by him as the first (the one currently in the bottom left) in such a way that it is easily seen as the first one - which he thinks can be done by moving it to the "top" location. 


\section{Transcript 7.1}

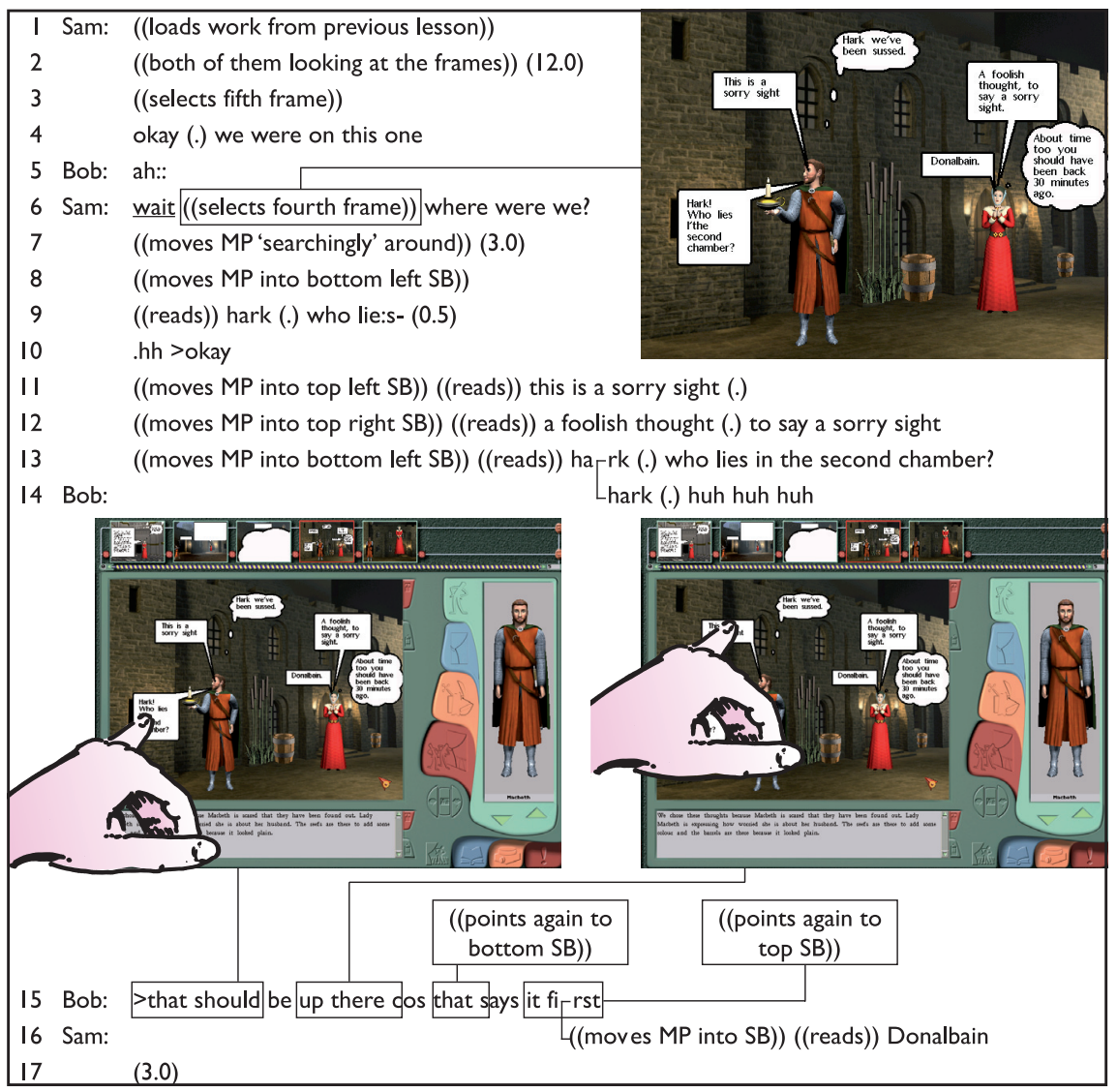

Sam does not immediately react to this, but instead finishes going-throughthe-speech-bubbles, reading out the final speech bubble: "Donalbain" (line 16). He then pauses and releases the mouse (line 17).

Sam now points to the screen and says: "no: (.) actually that [pointing at the bottom left speech bubble] goes first" (Transcript 7.2; line 18) to which Bob quickly agrees (line 19). In other words, Sam in line 18 produces another self-correction of his suggested reading order: it is not Macbeth's "top" speech bubble ("This is a sorry sight") but rather the "left" speech bubble ("Hark! ...”) which comes first in the dialogue ("I've just remembered," line 18). Sam then proceeds to read out the bottom right speech bubble (lines 18/19) and the top left speech bubble (line 20). Having now found an acceptable reading order, Sam takes control of the mouse, 
Transcript 7.2

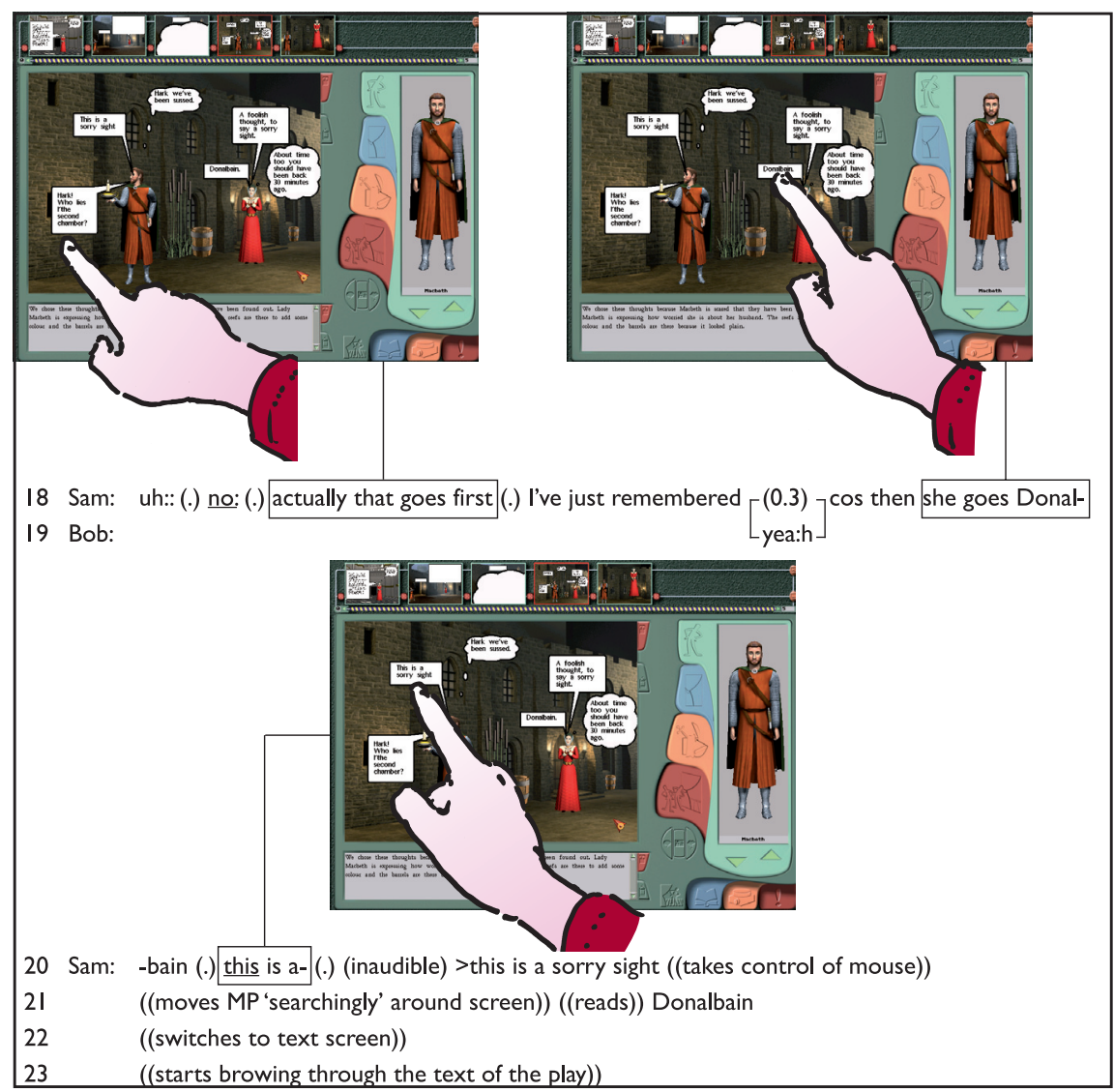

switches to the text screen (line 22), and starts to search for the piece of text that they will put in the next frame (line 23).

What is noteworthy about this episode is that it displays a clear ambiguity in the reading path of the speech bubbles, where this is an ambiguity for the pupils who have problems figuring out which speech bubble comes first in the dialogue. Furthermore, this is an ambiguity in a frame that they have constructed themselves (i.e., not an example of "interpreting the work of others"). There are two candidates: the top left speech bubble ("This is a sorry sight") and the bottom left one ("Hark! ...”), where, as mentioned above, the former would be first under the top-down rule, while the latter would be first under the left-right rule (see McCloud's suggested reading path in Figure 5). Sam has three attempts of identifying a reading order: 
- $\quad$ he starts by choosing the bottom left speech bubble as the first (line 9);

- he then self-corrects to choose a different path, starting with the top left speech bubble (line 11), moving to top right (line 12), bottom left (line 13), bottom right (line 16);

- after Bob's interruption (line 15), Sam corrects himself once more, starting with the bottom left speech bubble (line 18), then bottom right (lines 19/20) and then top left (line 22).

In contrast to Sam, Bob is not just concerned with finding the first speech bubble, but also with how this could be indicated within the frame. In other words, once they have agreed on which speech bubble is first, where should that speech bubble be placed? Bob thus thinks that the current ambiguity in the reading path could be removed (by moving Macbeth's speech bubbles).

What is interesting in this respect is Sam's "no" in his utterance "no (.) actually that goes first" (line 18). The "no" suggests a disagreement with Bob, but it is unclear what this is a disagreement about. Does Sam think that they disagree over which speech bubbles comes first in the dialogue, or do they disagree about the location of that speech bubble in the frame. What seems clear from Bob's reaction to Sam ("yeah," line 19) is that at least for Bob it is not a disagreement about which speech bubble comes first (Bob's reaction could be heard as "yeah, that's what I said"), but rather that Bob thinks that this speech bubble should therefore be moved to the top. In any case, this disagreement does not become topicalized. Sam does not ask Bob why he wants to move the left speech bubble "up there" - but neither does Bob repeat his proposal with an alternative explanation.

\section{Conclusion}

The aim of this paper has been to open up the "black box" of assembly procedures in the production of visual objects in order to begin to see the role that conventions play when participants are in the process of designing particular objects. In that sense, the goal has been to extend the work of researchers such as Kress and van Leeuwen (2006 [1996]) by moving the investigation of the visual grammar of objects from an analysis based on (finished) products to the processes involved in assembling those products (cf. Burn and Parker 2001: 159). This has been done by drawing on a different line of research, video analysis of naturally occurring interaction, which studies how participants orient to each other during interaction. 
By basing the analysis on video fragments in which pupils are in the process of constructing their storyboards has enabled this study to address the problem which arises in studies based solely on products, namely, that the design rationale is not always recoverable from the finished object, but may have to be stipulated by the researchers. For example, both Forceville (1999) and Bateman et al. (2004) have questioned aspects of Kress and van Leeuwen's analysis on the basis that "it has not yet been established whether such an analysis is actually any more than a post hoc rationalization of design decisions that occur on a page for quite other reasons" (Bateman et al. 2004: 67). In order to avoid the danger of such "post hoc analysis," it would be necessary to access the reasons and rationales during the design of objects. Video recordings of design-in-interaction allow us to do just that.

However, as outlined in the introduction, this is by no means straightforward. First, not every setting lends itself to being recorded. What is needed are "perspicuous settings" (Garfinkel 2002: 141) in which participants work together and therefore formulate for each other the reasons for their design. Second, even in such settings a lot of data may be required in order to find instances in which design decisions are explicitly topicalized. For example, this paper has been based on a rather modest number of instances (four in total). However, in each of these it could be shown that the pupils themselves discussed an aspect of the visual composition of their storyboard.

The focus on process also entails new challenges in transcription. Obviously, there are different ways in which such activities can be transcribed (cf. Jewitt 2006: 38). This paper has tried to reproduce what pupils said, what was displayed on the computer screen in front of them, and how pupils used their bodies to point to or visualize what they were saying.

The substantive focus of the analysis has been on how pupils arranged the speech bubbles in their visual representation of Shakespeare's play. It was shown that in contrast to comics, where there are relatively strong conventions that indicate a clear "reading path" for the order in which speech bubbles should be read (in particular, to indicate which speech bubble should be read first), pupils in this setting were for the most part not particularly concerned with indicating a "reading path." In fact, the placement of speech bubbles was for the most part a "nondecision decision," i.e., done without much deliberation or discussion. The speech bubbles were predominantly simply placed above or next to the character to which they were attached (which is where they first appeared on inserting them). In only a very few instances did pupils topicalize the reading order for speech bubbles. They did this by:

- numbering the speech bubbles (a strategy that was not sanctioned by the teacher); 
- moving the character who spoke first from the right side of the frame to the left (thereby simultaneously orienting to a left-to-right reading convention and the convention to place a speech bubble above a character's head); and

- placing speech bubbles in a column, one below the other (thereby making sure that only one rule, top-down, was in operation).

What is noteworthy is that in these instances it was only one of the two pupils who problematized the location of the speech bubbles (i.e., saw a problem), while the other pupil did not seem, particularly, to care (i.e., did not see a problem). For example, in Fragment 5, Sam wanted to move Macbeth from the right to the left, since it is Macbeth who speaks first, while Bob did not seem to see why this might be important (line 28: “yeah?”). In contrast, in Fragment 7, it is Bob who was keen to change the placement of the speech bubbles, while Sam remained unresponsive to Bob's suggestions. It was thus not always the same pupil who was concerned with this question. In other words, it was not that there was a difference between the pupils in terms of competence or knowledge.

This may be a result of the fact that the participants in this setting were not experts, but novices. There are, as Kress (2003: 115) has remarked, methodological advantages in observing "unpracticed designers," since we can see what they treat as problematic or strange. However, what this paper also shows is that notions of "grammar" or "convention" are only operating in a weak sense. It was not only that pupils were in some sense still learning the conventions underlying the placement of speech bubbles, but also that this was not necessarily their focus. Their storyboard was supposed to exhibit their understanding of Shakespeare's text and although the visual composition could contribute to that, it was predominantly the written text (in the thought bubbles and caption box) that the teacher oriented to when reviewing their work.

\section{References}

Amerine, R. \& J. Bilmes. 1988. Following instructions. Human Studies 11 (2-3). 327-339.

Anstey, M. \& G. Bull. 2000. Reading the visual: Written and illustrated children's literature. Sydney: Harcourt.

Atkinson, J. M. \& J. Heritage (eds.). 1984. Structures of social action: Studies in conversation analysis. Cambridge: Cambridge University Press.

Banks, M. 2001. Visual methods in social research. London: Sage.

Bateman, J., J. Delin \& R. Henschel. 2004. Multimodality and empiricism: Preparing for a corpus-based approach to the study of multimodal meaning-making. In E. Ventola, C. Charles \& M. Kaltenbacher (eds.), Perspectives on multimodality, 65-87. Amsterdam: John Benjamins. 
Bennerstedt, U. 2008. Welcome to the digital puppet show: Positioning work and make-believe methods in role play Mmorpg servers. In O. Leino, G. Calleja \& S. Mosberg Iversen (eds.), Proceedings of the [Player] conference, Copenhagen, August 26-29, 53-86. Copenhagen: Nørrebros Bogtryk ApS.

Broth, M. 2008. The "listening shot" as a collaborative practice for categorizing studio participants in a live TV-production. Ethnographic Studies 10. 69-88.

Broth, M. 2009. Seeing through screens, hearing through speakers: Managing distant studio space in television control room interaction. Journal of Pragmatics 41(10). 1998-2016.

Brown, B. \& M. Bell. 2004. CSCW at play: "There” as a collaborative virtual environment. In Proceedings of CSCW'04, Chicago, November 6-10, 350-359. New York: ACM Press.

Burn, A. \& D. Parker. 2001. Making your mark: Digital inscription, animation, and a new visual semiotic. Education, Communication \& Information 1(2). 155-179.

Büscher, M. 2005. Social life under the microscope? Sociological Research Online 10(1). http://www.socresonline.org.uk/10/1/buscher.html (accessed 15 September 2012).

Coles, J. 2009. Testing Shakespeare to the limit: Teaching "Macbeth" in a Year 9 classroom. English in Education 43(1). 32-49.

Eisner, W. 2008 [1985]. Comics \& sequential art. New York, NY: W. W. Norton.

Emmison, M. \& P. Smith. 2000. Researching the visual: Images, objects, contexts and interactions in social and cultural inquiry. London: Sage.

Engström, A., O. Juhlin, M. Perry \& M. Broth. 2010. Temporal hybridity: Mixing live video footage with instant replay in real time. In Proceedings of CHI 2010, Atlanta, 1495-1504. New York: ACM Press.

Evans, J. \& S. Hall (eds.). 1999. Visual culture: The reader. London: Sage.

Forceville, C. 1999. Educating the eye? Kress and Van Leeuwen's Reading images: The grammar of visual design (1996). Language and Literature 8(2). 163-178.

Franks, A. 2003. Palmer's kiss: Shakespeare, school drama and semiotics. In C. Jewitt \&

G. Kress (eds.), Multimodal literacy, 155-172. New York: Peter Lang.

Garfinkel, H. 1967. Studies in ethnomethodology. Englewood Cliffs, NJ: Prentice-Hall.

Garfinkel, H. 2002. Ethnomethodology's program: Working out Durkheim's aphorism. Lanham, MD: Rowman \& Littlefield.

Gibson, R. 1998. Teaching Shakespeare. Cambridge: Cambridge University Press.

Goffman, E. 1979. Gender advertisements. London: Macmillan.

Goodwin, C. 1981. Conversational organization: Interaction between speakers and hearers. New York: Academic Press.

Goodwin, C. 1986. Gestures as a resource for the organization of mutual orientation. Semiotica 62(1/2). 29-49.

Goodwin, C. 1994. Professional vision. American Anthropologist 96(3). 606-633.

Goodwin, C. 2001. Practices of seeing visual analysis: An ethnomethodological approach. In T. van Leeuwen \& Carey Jewitt (eds.), Handbook of visual analysis, 157-182. London: Sage.

Goody, J. 1977. The domestication of the savage mind. Cambridge: Cambridge University Press.

Greiffenhagen, C. 2008. Unpacking tasks: The fusion of new technology with instructional work. Computer Supported Cooperative Work 17(1). 35-62.

Greiffenhagen, C. 2012. Making rounds: The routine work of the teacher during computersupported collaborative learning. International Journal of Computer-Supported Collaborative Learning 7(1). 11-42. 
Greiffenhagen, C. \& R. Watson 2009. Visual repairables: Analyzing the work of repair in human-computer interaction. Visual Communication 8(1). 65-90.

Haladewicz-Grzelak, M. 2009. Visual syntax in the iconography of Saint Nicholas. Semiotica 176(1/4). 131-164.

Hall, S. 1972. The determinations of news photographs. Working Papers in Cultural Studies 3. 53-87.

Heath, C. 1982. The display of recipiency: An instance of a sequential relationship in speech and body movement. Semiotica 42(2/4). 147-167.

Heath, C. 1986. Body movement and speech in medical interaction. Cambridge: Cambridge University Press.

Heath, C., M. Jirotka, P. Luff \& J. Hindmarsh. 1995. Unpacking collaboration: The interactional organization of trading in a city dealing room. Computer Supported Cooperative Work 3(2). 147-165.

Heath, C. \& P. Luff. 1992. Collaboration and control: Crisis management and multimedia technology in London underground line control rooms. Computer Supported Cooperative Work 1(1-2). 69-94.

Heath, C., J. Hindmarsh \& P. Luff. 2010. Video in qualitative research: Analyzing social interaction in everyday life. London: Sage.

Heritage, J. 2002. The limits of questioning: Negative interrogatives and hostile question content. Journal of Pragmatics 34(10-11). 1427-1446.

Horn, R. E. 1998. Visual language: Global communication for the twenty-first century. Bainbridge Island, WA: MacroVU.

Ivarsson, J. 2010. Developing the construction sight: Architectural education and technological change. Visual Communication 9(2). 171-191.

Jayyusi, L. 1988. Toward a socio-logic of the film text. Semiotica 68(3/4). 271-296.

Jewitt, C. 2002. The move from page to screen: The multimodal reshaping of school English. Visual Communication 1(2). 171-195.

Jewitt, C. 2006. Technology, literacy, and learning: A multimodal approach. London: Routledge.

Knox, J. 2007. Visual-verbal communication on online newspaper home pages. Visual Communication 6(1). 19-53.

Koshik, I. 2002. A conversation analytic study of yes/no questions which convey reversed polarity assertions. Journal of Pragmatics 34(12). 1851-1877.

Kress, G. 2003. Literacy in the New Media age. London: Routledge.

Kress, G. 2010. Multimodality: A social semiotic approach to contemporary communication. London: Routledge.

Kress, G., C. Jewitt, J. Bourne, A. Franks, J. Hardcastle, K. Jones \& E. Reid. 2005. English in urban classrooms: A multimodal perspective on teaching and learning. London: Routledge.

Kress, G. \& T. van Leeuwen. 2006 [1996]. Reading images: The grammar of visual design, $2^{\text {nd }}$ edn. London: Routledge.

Laurier, E. \& B. Brown. 2011. The reservations of the editor: The routine work of showing and knowing the film in the edit suite. Social Semiotics 21(2). 239-257.

Madden, M., P. W. Chung \& C. W. Dawson. 2009. Cartoons beyond clipart: A computer tool for storyboarding and storywriting. Computers \& Education 52(1). 188-200.

Mahin, D. 2007. Introduction to multimodal analysis. London: Hodder Arnold.

Maiorani, A. 2007. "Reloading" movies into commercial reality: A multimodal analysis of The Matrix trilogy's promotional posters. Semiotica 166(1/4). 45-67.

McCloud, S. 1994. Understanding comics. New Yorker: HarperCollins. 
McCloud, S. 2006. Making comics: Storytelling secrets of comics, manga and graphic novels. New York: HarperCollins.

Mirzoeff, N. 2009 [1999]. An introduction to visual culture, $2^{\text {nd }}$ edn. London: Routledge.

Mondada, L. 2003. Working with video: How surgeons produce video records of their actions. Visual Studies 18(1). 58-73.

Mondada, L. 2007. Multimodal resources for turn-taking: Pointing and the emergence of possible next speakers. Discourse Studies 9(2). 195-226.

Morrison, K. L. 1981. Some properties of "telling-order designs" in didactic inquiry. Philosophy of the Social Sciences 11(2). 245-262.

Murphy, K. M. 2005. Collaborative imagining: The interactive use of gestures, talk, and graphic representation in architectural practice. Semiotica 156(1/4). 113-145.

O'Halloran, K. 1999. Towards a systemic functional analysis of multisemiotic mathematics texts. Semiotica 124(1/2). 1-29.

Pink, S. 2006. The future of visual anthropology: Engaging the senses. London: Routledge.

Rose, G. 2007 [2001]. Visual methodologies: An introduction to the interpretation of visual materials, $2^{\text {nd }}$ edn. London: Sage.

Sacks, H. 1992. Lectures on conversation, G. Jeeferson (ed.). Oxford: Blackwell.

Schegloff, E. A. 2007. Sequence organization in interaction. Cambridge: Cambridge University Press.

Sharrock, W. W. \& D. R. Watson. 1985. "Reality construction” in L2 simulations. System 13(3). 195-206.

Stanczak, G. (ed.). 2007. Visual research methods: Image, society, and representation. London: Sage.

Sturken, M. \& L. Cartwright. 2001. Practices of looking: An introduction to visual culture. Oxford: Oxford University Press.

van Leeuwen, T. \& C. Jewitt (eds.). 2001. Handbook of visual analysis. London: Sage.

Watson, D. R. 1997. Ethnomethodology and textual analysis. In D. Silverman (ed.), Qualitative research: Theory, method, and practice, 80-98. London: Sage.

Williamson, J. 1978. Decoding advertisements: Ideology and meaning in advertising. London: Martin Boyars.

Yannicopoulou, A. 2004. Visual aspects of written texts: Preschoolers view comics. Educational Studies in Language and Literature 4(2-3). 169-181. 


\section{Appendix 1}

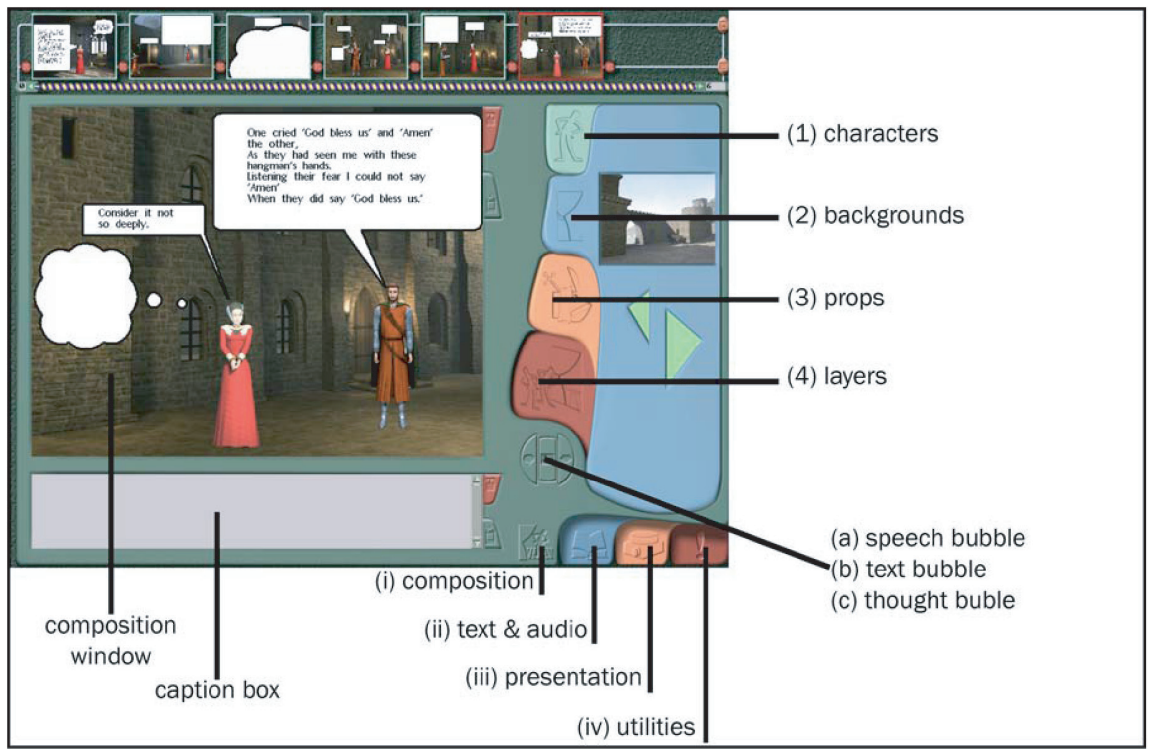

Fig. 13: The interface of the software

\section{Appendix 2 The beginning of Act 2, Scene 2}

\section{LADY MACBETH}

2 That which hath made them drunk hath made me hold;

3 What hath quenched them hath given me fire.-Hark!-Peace!

4 It was the owl that shrieked, the fatal bellman

5 Which gives the stern'st good-night. He is about it.

6 The doors are open, and the surfeited grooms

7 Do mock their charge with snores; I have drugged their possets

8 That death and nature do contend about them

9 Whether they live or die.

11 MACBETH

12 Who's there? What, ho! 
14 LADY MACBETH

15 Alack, I am afraid they have awaked,

16 And 'tis not done. The attempt and not the deed

17 Confounds us. -Hark!- I laid their daggers ready;

18 He could not miss'em. Had he not resembled

19 My father as he slept, I had done't.

20

21 My husband!

22

23 MACBETH

24 I have done the deed. Dist thou not hear a noise?

25

26 LADY MACBETH

27 I heard the owl-scream and the cricket's cry.

28 Did not you speak?

29

30 MACBETH

31 When?

32

33 LADY MACBETH

34 Now.

35

36 MACBETH

37 As I descended?

38

39 LADY MACBETH

40 Ay.

41

42 MACBETH

43 Hark!

44 Who lies I'the second chamber?

45

46 LADY MACBETH

47 Donalbain.

48

49 MACBETH

50 This is a sorry sight

51

52 LADY MACBETH

53 A foolish thought, to say a sorry sight. 
54

55 MACBETH

56 There's one did laugh in's sleep, and one cried "Murder!"

57 That they did wake each other. I stood and heard them.

58 But they did say their prayers and addressed them

59 Again to sleep.

60

61 LADY MACBETH

62 There are two lodged together.

63

64 MACBETH

65 One cried "God bless us" and "Amen" the other,

66 As they had seen me with these hangman's hands.

67 Listening their fear I could not say "Amen"

68 When they did say "God bless us."

69

70 LADY MACBETH

71 Consider it not so deeply.

\section{Bionote}

Christian Greiffenhagen (b. 1973) is a Research Fellow at the University of Nottingham 〈christian.greiffenhagen@nottingham.ac.uk〉. His research interests include science \& technology studies (STS), human-computer interaction (HCI), video analysis and visual studies, and philosophy of social science. His publications include "Video analysis of mathematical practice? Different attempts to 'open up' mathematics for sociological investigation" (2008); and "Mathematical relativism: Logic, grammar, and arithmetic in cultural comparison" (with W. Sharrock, 2009). 
